\title{
THE STATE OF THE GAS AND THE RELATION BETWEEN GAS AND STAR FORMATION AT LOW METALLICITY: THE SMALL MAGELLANIC CLOUD
}

\author{
Alberto D. Bolatto $^{1}$, Adam K. Leroy ${ }^{2,13}$, Katherine Jameson ${ }^{1}$, Eve Ostriker ${ }^{1}$, Karl Gordon ${ }^{3}$, Brandon Lawton ${ }^{3}$, \\ Snežana Stanimirović ${ }^{4}$, Frank P. IsRael ${ }^{5}$, SuZanne C. Madden ${ }^{6}$, Sacha HonY ${ }^{6}$, Karin M. Sandstrom ${ }^{7,14}$, \\ Caroline Bot ${ }^{8}$, Mónica Rubio ${ }^{9}$, P. Frank Winkler ${ }^{10}$, Julia Roman-Duval ${ }^{3}$, Jacco Th. van Loon ${ }^{11}$, \\ JOANA M. OLIVEIRA ${ }^{11}$, AND RÉMY INDEBETOUW ${ }^{2,12}$ \\ ${ }^{1}$ Department of Astronomy and Laboratory for Millimeter-wave Astronomy, University of Maryland, \\ College Park, MD 20742, USA; bolatto@astro.umd.edu \\ 2 National Radio Astronomy Observatory, Charlottesville, VA 22903, USA \\ ${ }^{3}$ Space Telescope Science Institute, Baltimore, MD 21218, USA \\ ${ }^{4}$ Department of Astronomy, University of Wisconsin, Madison, WI 53706, USA \\ ${ }^{5}$ Leiden Observatory, Leiden, NL-2300 RA, The Netherlands \\ ${ }^{6}$ Laboratoire AIM Paris-Saclay, CNRS/INSU CEA/Irfu Université Paris Diderot, 91191 Gif sur Yvette, France \\ ${ }^{7}$ Max-Planck-Institut für Astronomie, D-69117 Heidelberg, Germany \\ ${ }^{8}$ Observatoire Astronomique de Strasbourg, Université de Strasbourg, and CNRS, 67000 Strasbourg, France \\ ${ }^{9}$ Departamento de Astronomía, Universidad de Chile, Casilla 36-D, Santiago, Chile \\ ${ }^{10}$ Department of Physics, Middlebury College, Middlebury, VT 05753, USA \\ ${ }^{11}$ Lennard-Jones Laboratories, Keele University, ST5 5BG, UK \\ ${ }^{12}$ Department of Astronomy, University of Virginia, Charlottesville, VA 22904, USA \\ Received 2011 April 15; accepted 2011 July 3; published 2011 October 11
}

\begin{abstract}
We compare atomic gas, molecular gas, and the recent star formation rate (SFR) inferred from H $\alpha$ in the Small Magellanic Cloud (SMC). By using infrared dust emission and local dust-to-gas ratios, we construct a map of molecular gas that is independent of $\mathrm{CO}$ emission. This allows us to disentangle conversion factor effects from the impact of metallicity on the formation and star formation efficiency of molecular gas. On scales of 200 pc to $1 \mathrm{kpc}$ (where the distributions of $\mathrm{H}_{2}$ and star formation match well) we find a characteristic molecular gas depletion time of $\tau_{\text {dep }}^{\text {mol }} \sim 1.6 \mathrm{Gyr}$, similar to that observed in the molecule-rich parts of large spiral galaxies on similar spatial scales. This depletion time shortens on much larger scales to $\sim 0.6$ Gyr because of the presence of a diffuse $\mathrm{H} \alpha$ component, and lengthens on much smaller scales to $\sim 7.5 \mathrm{Gyr}$ because the $\mathrm{H} \alpha$ and $\mathrm{H}_{2}$ distributions differ in

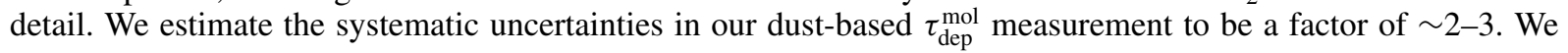
suggest that the impact of metallicity on the physics of star formation in molecular gas has at most this magnitude, rather than the factor of $\sim 40$ suggested by the ratio of SFR to CO emission. The relation between SFR and neutral $\left(\mathrm{H}_{2}+\mathrm{H}\right.$ I) gas surface density is steep, with a power-law index $\approx 2.2 \pm 0.1$, similar to that observed in the outer disks of large spiral galaxies. At a fixed total gas surface density the SMC has a 5-10 times lower molecular gas fraction (and star formation rate) than large spiral galaxies. We explore the ability of the recent models by Krumholz et al. and Ostriker et al. to reproduce our observations. We find that to explain our data at all spatial scales requires a low fraction of cold, gravitationally bound gas in the SMC. We explore a combined model that incorporates both large-scale thermal and dynamical equilibrium and cloud-scale photodissociation region structure and find that it reproduces our data well, as well as predicting a fraction of cold atomic gas very similar to that observed in the SMC.
\end{abstract}

Key words: galaxies: dwarf - galaxies: evolution - ISM: clouds - Magellanic Clouds

Online-only material: color figures

\section{INTRODUCTION}

The relation between gas content and star formation activity in galaxies has been a matter of intense investigation since the pioneering work of Maarten Schmidt. Schmidt (1959) suggested that the star formation rate (SFR) in a galaxy is proportional to a power of the gas density, such that $\rho_{\mathrm{SFR}} \sim \rho_{\text {gas }}^{n}$, where $n \simeq 1-3$ and most likely $n=2$ based on a number of arguments that included the luminosities of open clusters, the abundance of helium, and the vertical distribution of objects in the plane of the Milky Way. Here we refer to the quantitative relation between gas and star formation as the "star formation law" for convenience without intending to suggest a physical law or a specific functional form.

\footnotetext{
${ }^{13}$ Hubble Fellow.

${ }^{14}$ Marie Curie Fellow.
}

Most modern empirical studies of the extragalactic star formation law follow those by Kennicutt $(1989,1998)$, who studied disk-averaged correlations in a sample of $\sim 100$ galaxies including starbursts and high-mass dwarfs. This work linked the surface density of SFR, $\Sigma_{\mathrm{SFR}}$, to the surface density of total neutral (atomic and molecular) gas, $\Sigma_{\mathrm{gas}}=\Sigma_{\mathrm{H} \mathrm{I}}+\Sigma_{\mathrm{H} 2}$. Kennicutt (1998) found that $\Sigma_{\mathrm{SFR}} \propto \Sigma_{\mathrm{gas}}{ }^{1+p}$ with $1+p \approx 1.4 \pm 0.15$ for his composite sample of galaxies. Since the gas depletion time $\tau_{\text {dep }}^{\text {gas }} \equiv \Sigma_{\text {gas }} / \Sigma_{\text {SFR }} \propto \Sigma_{\text {gas }}^{-p}$ for a power-law relation, $p=0$ indicates that the gas depletion time is constant (independent of environment), while $p>0$ indicates that star formation is more rapid in high- $\Sigma_{\text {gas }}$ regions. In practice, it is also important to keep in mind the observational complexities associated with measuring $\Sigma_{\text {gas }}$ and particularly $\Sigma_{\mathrm{H} 2}$ from CO observations, and the systematics thus introduced. 
Several subsequent studies focused on the star formation law within galaxies, employing high-resolution molecular and $\mathrm{H}$ I observations (Martin \& Kennicutt 2001; Wong \& Blitz 2002; Boissier et al. 2003). More recently, Bigiel et al. (2008) and Leroy et al. (2008) analyzed 12 nearby spirals with high-quality $\mathrm{H}$, CO, far-infrared, and far-ultraviolet data. These observations show a clear correlation between $\Sigma_{\mathrm{SFR}}$ and the surface density of molecular gas, $\Sigma_{\mathrm{H} 2}$, with an approximately constant SFR per unit molecular gas, yielding an approximately linear power-law index $1+p=1.0 \pm 0.2$. By contrast, they found a steep correlation between $\Sigma_{\mathrm{SFR}}$ and the surface density of atomic gas, $\Sigma_{\mathrm{HI}}$ (the observed correlation in the optical disks of large galaxies has $1+p \gtrsim 2.7-3.5$ and $1+p \sim 2$ in the H I-dominated outer disks; Bigiel et al. 2008, 2010b). Schruba et al. (2011) and Bigiel et al. (2011) extended these findings to a larger sample of 30 disk galaxies, while Blanc et al. (2009) and Rahman et al. (2011) arrived at similar conclusions in very detailed studies of individual targets.

A consequence of the observed linearity of the star formation law over the molecule-dominated regions of disks is that the typical timescale to deplete the molecular gas by star formation in a disk galaxy is $\tau_{\mathrm{dep}}^{\mathrm{mol}}=\Sigma_{\mathrm{mol}} / \Sigma_{\mathrm{SFR}} \sim 1.9 \pm 0.9 \mathrm{Gyr}\left(\Sigma_{\mathrm{mol}}\right.$ corresponds to $\Sigma_{\mathrm{H} 2}$ corrected by a factor of 1.36 to account for the cosmic abundance of helium). The lack of dependence of $\tau_{\mathrm{dep}}^{\mathrm{mol}}$ on environment can be naturally understood if two conditions are fulfilled. The first condition is for star formation to be a local process primarily determined by the conditions inside the giant molecular clouds (GMCs) where it takes place. GMCs are themselves isolated from the global galactic environment provided that they are self-gravitating and therefore overpressured with respect to the neighboring gas (McKee \& Ostriker 2007). The second condition is for the properties of GMCs to be universal, and therefore independent of their galactic environment. Because star formation takes place in the densest regions of GMCs, themselves self-gravitating and thus mostly decoupled from their surroundings, the first condition appears likely (Krumholz \& McKee 2005), at least in mid-disk and outer-galaxy regions. In galactic centers it is less clear whether there are isolated, gravitationally bound GMCs or instead a distributed molecular medium. Environmental considerations may be more important if cloud-cloud collisions play an important role (Tan 2000). Similarly, the second condition appears to be broadly satisfied as the resolved properties of GMCs (or at least of the dense regions of GMCs, bright in $\mathrm{CO}$ ) are observed to be remarkably constant over a wide range of galaxy environments (the Local Group spirals and nearby dwarfs, Bolatto et al. 2008; the Milky Way, Heyer et al. 2009; the LMC, Hughes et al. 2010; the outer disk of M33, Bigiel et al. 2010a).

Most of the studies of the relation between gas and star formation, and the ensuing conclusions, focus on large galaxies which tend to be rich in molecules. There is a dearth of comparable information for low-mass low-metallicity star-forming dwarf galaxies, primarily because such objects emit only very faintly in molecular gas tracers such as $\mathrm{CO}$. The measurements that do exist reveal large ratios of SFR to $\mathrm{CO}$ emission in latetype, low-mass galaxies (e.g., Young et al. 1996; Gardan et al. 2007; Leroy et al. 2007b; Krumholz et al. 2011).

Studies of the star formation law in dwarf galaxies are interesting for a number of reasons. Fundamentally they probe a very different physical regime from large spiral galaxies, one where atomic gas dominates the surface density of the interstellar medium (ISM) on large scales, and elements heavier than helium are less abundant. This dearth of heavy elements causes a number of differences in gas chemistry and physical conditions. For example, dust-to-gas ratios are lower, producing lower extinctions and higher photodissociation rates (the likely cause of their general CO faintness; Israel et al. 1986; Maloney \& Black 1988; Lequeux et al. 1994; Bolatto et al. 1999).

More importantly, studies of the star formation law in lowmass low-metallicity star-forming dwarf galaxies provide fertile testing ground for star formation theories. A natural implication of the approximately constant molecular $\tau_{\mathrm{dep}}^{\mathrm{mol}}$ among normal galaxies is that the effectiveness at forming molecular gas plays a critical role in establishing the SFR. The molecular gas fraction varies systematically both within and among galaxies (Young \& Scoville 1991; Wong \& Blitz 2002; Blitz \& Rosolowsky 2006; Leroy et al. 2008). Dwarf galaxies appear to have low molecular fractions, despite their large gas fractions and long timescales to deplete gas reservoirs at current rates of star formation. If their $\tau_{\text {dep }}^{\text {mol }}$ values are similar to normal galaxies, it would suggest that GMC formation (and destruction) is the rate-limiting step for star formation in dwarf systems.

Because their low metallicities contrast with those in spiral galaxies, star-forming dwarf galaxies can break a number of degeneracies in the physical drivers of the molecular fraction. Different models predict very different behaviors for the impact of metallicity on molecular fractions and SFRs according to their emphasis on dynamics, thermodynamic equilibrium, or dust shielding. For example, the requirement of a minimum extinction for star formation to occur (e.g., McKee 1989; Lada et al. 2009) would have a proportionally more important impact in low-metallicity objects, while models that are solely based on dynamics cannot distinguish between low and high metallicity.

The main obstacle to using dwarf galaxies to test various aspects of the star formation law is the difficulty in observing their molecular gas distribution, stemming from the faintness of their $\mathrm{CO}$ emission, and the uncertainty in its quantitative relation to $\mathrm{H}_{2}$. The Small Magellanic Cloud (SMC) offers a unique opportunity in this regard. Its proximity allows us to probe small spatial scales with observations of modest angular resolution. As a result, we can use dust emission to construct a map of $\Sigma_{\mathrm{H} 2}$ that does not depend on CO. In this study we use such a map to compare the distributions of molecular gas, atomic gas, and recent star formation traced by ionizing photon production.

\subsection{The Small Magellanic Cloud as a Galaxy}

By virtue of its location and properties the SMC is a prime laboratory for the study of the relation between gas and star formation in low-mass galaxies. Indeed, the SMC was the target of the first extragalactic study of the star formation law, relating H I and stellar surface densities (Sanduleak 1969). Located scarcely $61 \mathrm{kpc}$ away (Hilditch et al. 2005; Keller \& Wood 2006; Szewczyk et al. 2009), the SMC is the nearest gasrich low-metallicity dwarf galaxy with active star formation $\left(Z_{\mathrm{SMC}} \sim Z_{\odot} / 5\right.$; Dufour 1984; Kurt et al. 1999; Pagel 2003). As such, it provides invaluable insight into the physics and chemistry of the ISM in chemically primitive star-forming galaxies. Moreover, because of its proximity it is possible to carry out studies on the scale of individual young stellar objects, which reveal subtle differences in the temperature and chemistry of star-forming cores (van Loon et al. 2010; Oliveira et al. 2011).

With an $\mathrm{H}_{\mathrm{I}}$ mass of $M_{\mathrm{HI}} \simeq 4.2 \times 10^{8} M_{\odot}$ (Stanimirović et al. 1999) the SMC is rich in atomic gas, although also strikingly 
deficient in cold H I (Dickey et al. 2000). It was already apparent in the early observations that the distribution of the $\mathrm{HI}_{\mathrm{I}}$ is complex, with more than one component along the line of sight (McGee \& Newton 1981). This complexity is at least in part due to the presence of several supergiant shells (Stanimirović et al. 1999). The underlying dynamics appear to be disk-like with an inclination $i \approx 40^{\circ} \pm 20^{\circ}$, although disturbed by the interaction with the Milky Way and the LMC (Stanimirović et al. 2004). In our analysis we will ignore this complexity, but the geometry of the SMC and the level of turbulent support in the gas remain some of the most important caveats in the comparison to star formation models.

The SMC hosts a healthy amount of star formation despite being disproportionately faint in $\mathrm{CO}$ emission (Kennicutt et al. 1995; Israel et al. 1986, 1993). In fact, in the SMC the CO is underluminous with respect to the star formation activity by almost two orders of magnitude when compared to normal disks, or even more massive small galaxies. A possible explanation for this fact is that the SMC is extraordinarily efficient at turning the available molecular gas into stars (i.e., the $\mathrm{H}_{2}$ depletion time is very short), which would suggest that we are either observing an out-of-equilibrium situation (a fleeting starburst) or that the conditions conspire to keep a small reservoir of extremely shortlived GMCs. A more likely alternative is that the weak CO emission is not representative of star-forming molecular gas, with $\mathrm{H}_{2} / \mathrm{CO}$ considerably larger than in the Milky Way (Israel et al. 1986; Rubio et al. 1993).

Leroy et al. (2007a) used new far-infrared observations to map the $\mathrm{H}_{2}$ distribution in the SMC bypassing $\mathrm{CO}$ emission, following an extension of the methodology previously employed by Israel (1997) in the Magellanic Clouds. The feasibility of using dust-to-map $\mathrm{H}_{2}$ was clearly demonstrated by Dame et al. (2001) in the Milky Way (see also Bloemen et al. 1990 for an early study of the correlation between dust emission and gas in the Milky Way). It was also shown to be consistent with virial masses on large scales (Leroy et al. 2009). Furthermore, a systematic application of an extension of this methodology throughout the Local Group produces molecular masses that are consistent with those obtained by other methods at approximately Galactic metallicities (Leroy et al. 2011). The analysis by Leroy et al. (2007a) shows that the column density of molecular gas present in the SMC is far in excess of that derived by applying the Galactic $\mathrm{CO}$-to- $\mathrm{H}_{2}$ conversion factor, $X_{\mathrm{CO}}$, to the CO observations. Their modeling yields a total molecular mass $M_{\mathrm{H} 2} \approx 3.2 \times 10^{7} M_{\odot}$. Therefore, the neutral ISM in the SMC is approximately $10 \%$ molecular, a lower fraction than observed in normal late spirals but not dramatically so (Young \& Scoville 1991). Further refinements to the analysis (Leroy et al. 2009, 2011; and this work) broadly confirm these numbers.

In this study we analyze the spatially resolved correlation between the distributions of molecular gas, atomic gas, and recent star formation traced by ionizing photon production on different scales, comparing the star formation law in this small low-metallicity galaxy with that in large disks. We present our data and discuss the methodology we use to measure molecular column densities and SFRs in Section 2. We show our main observational results in Section 3, focusing on the relation between $\mathrm{H}_{2}$ and star formation in Section 3.2, and total gas and star formation in Section 3.3. In Section 4, we compare our results to recent analytical physical models of star formation in galaxies. Finally, we summarize our conclusions in Section 5 .

\section{OBSERVATIONS AND METHODOLOGY}

\subsection{Estimating $\mathrm{H}_{2}$ from Infrared Dust Emission}

To estimate molecular gas surface densities from far-infrared emission, we use an extension of the methodology already employed by Leroy et al. (2009). Here we summarize the main points, but the reader should refer to that paper for the details as well as a more thorough discussion of the effects of several systematics involved in the derivation of the $\mathrm{H}_{2}$ map.

We combine $\mathrm{H}$ I and infrared (IR) imaging to estimate the distribution of $\mathrm{H}_{2}$ at a resolution $\theta \sim 40^{\prime \prime}(r \sim 12 \mathrm{pc})$. The IR data originate from the combination of two Spitzer surveys, SAGESMC (Gordon et al. 2011) and $S^{3} M C$ (Bolatto et al. 2007). The H I data are from Stanimirović et al. (1999) and include both interferometric (from the Australia Telescope Compact Array, ATCA) and single-dish (from Parkes) observations, and so are sensitive to emission on all spatial scales. When it is necessary to include an inclination correction we use $i \approx 40^{\circ}$ for the SMC, as derived from fitting the Hi kinematics (Stanimirović et al. 2004). Our analysis uses data from Spitzer's Multiband Imaging Photometer (MIPS) instrument at 70 and $160 \mu \mathrm{m}$. We correct the $160 \mu \mathrm{m}$ map for foreground contaminating emission from Galactic cirrus by subtracting the appropriately scaled $\mathrm{H}$ I emission from the Milky Way, which is identified on the basis of its velocities (see Bot et al. 2004; Leroy et al. 2009). Our gas surface densities include a $36 \%$ correction for the mass contribution of helium.

We derive the surface density of $\mathrm{H}_{2}, \Sigma_{\mathrm{H} 2}$, by modeling the IR emission to infer the amount of dust along each line of sight. Together with a dust-to-gas ratio (or strictly speaking a dust optical depth to $\mathrm{H} \mathrm{I}+\mathrm{H}_{2}$ surface density ratio), which we estimate by comparing dust and $\mathrm{H}_{\mathrm{I}}$ iteratively (see below), the dust emission allows us to estimate the total amount of gas present. Subtracting the measured H I surface density yields $\Sigma_{\mathrm{H} 2}$. We should caution that this procedure may identify as $\mathrm{H}_{2}$ very cold self-absorbed $\mathrm{H}$ I gas possibly associated with cloud envelopes, simply because this material will be disproportionally faint at $21 \mathrm{~cm}$ and thus incorrectly subtracted. We include a correction for optical depth that accounts for self-absorption in the $\mathrm{HI}$ map (Dickey et al. 2000), but such correction is by necessity only statistical. Thus, the procedure we outline may be more accurately thought of as producing a map of the very cold neutral and molecular phases, which under normal circumstances would be completely dominated by the mass of $\mathrm{H}_{2}$.

We use the optical depth at $160 \mu \mathrm{m}, \tau_{160}$, as our proxy for the amount of dust along the line of sight. We estimate this quantity following the method outlined by Leroy et al. (2009), though note that our calculations improve on theirs in several aspects. We take the ratio of IR intensities at 100 and $160 \mu \mathrm{m}$ $\left(I_{100}\right.$ and $\left.I_{160}\right)$ to be a tracer of the equilibrium temperature of a large grain population that contains most of the dust mass. Because the $100 \mu \mathrm{m}$ intensity comes from IRAS, we only have known it at $\sim 4^{\prime}$ resolution. To overcome this limitation to the resolution of the study we compare the $I_{100} / I_{160}$ ratio to the ratio of IR intensities at 70 and $160 \mu \mathrm{m}$. The emission at $70 \mu \mathrm{m}, I_{70}$, suffers from significant contamination by very small grains out of equilibrium, and it can only be used to determine the temperature of the large dust grains after removing the contamination. We calibrate a relationship that allows us to predict $I_{100} / I_{160}$ from $I_{70} / I_{160}$, at the coarse resolution of the $100 \mu \mathrm{m}$ data. The fact that the $I_{100} / I_{160}-I_{70} / I_{160}$ relation is well defined can be appreciated in Figure 2 in Leroy et al. (2009), and the fitted relations (Equations (2) and (3) in the same paper) 
suggest that half of $I_{70}$ is due to emission from stochastically heated grains. We apply our calibration at the full resolution of the $160 \mu \mathrm{m}$ data $\left(\sim 40^{\prime \prime}\right)$ to estimate the dust temperature and optical depth, $\tau_{160}$. Thus, this procedure allows us to take full advantage of the $160 \mu \mathrm{m}(\sim 12 \mathrm{pc})$ resolution while guaranteeing that we match the results derived from the 100 and $160 \mu \mathrm{m}$ on size scales larger than $70 \mathrm{pc}$.

We now have images of $\tau_{160}$, which linearly traces the distribution of dust, and the Hi column density from $21 \mathrm{~cm}$ radio observations corrected for optical depth effects (Dickey et al. 2000). To create an image of $\Sigma_{\mathrm{H} 2}$ we combine these maps to produce local estimates of our gas-to-dust ratio proxy, $\delta_{\mathrm{GDR}}=\Sigma_{\mathrm{gas}} / \tau_{160}$. Note that $\delta_{\mathrm{GDR}}$ is an observable in regions dominated by atomic gas. We use it because the actual value of the mass emissivity coefficient for dust is unimportant in producing the $\mathrm{H}_{2}$ map. Its systematic large-scale variations, however, matter and are very important to consider as we discuss below. Hence,

$$
\Sigma_{\mathrm{H} 2}=\tau_{160} \delta_{\mathrm{GDR}}-\Sigma_{\mathrm{HI}} .
$$

There are several ways to obtain the $\delta_{\mathrm{GDR}}$ from maps of dust and $\mathrm{H}_{\mathrm{I}}$. One could simply take the average ratio across the entire SMC, which is certainly $\mathrm{H}$ I dominated on large scales. Unfortunately, the SMC is known to harbor significant largescale variations in $\delta_{\mathrm{GDR}}$, with higher values in diffuse, lowdensity regions, and particularly in the SMC Wing (Stanimirović et al. 2000; Bot et al. 2004; Leroy et al. 2007a). Similar largescale variations, for example, appear to be present in the fraction of dust in polycyclic aromatic hydrocarbons (PAHs; Sandstrom et al. 2010) and in the optical extinction per gas column density (Dobashi et al. 2009) between lines of sight with dense and diffuse gas in the SMC. These variations are probably not rooted in variations in metallicity. Indeed, metallicity appears to be approximately uniform across the source (e.g., Dufour 1984), although note that these measurements are limited to the dense gas phases that form stars. Rather, they may be due to grain processing in the ISM. These variations could be analogous to the changes in dust optical depth per unit mass driven by temperature and grain structure changes observed over smaller scales in the Milky Way (Bernard et al. 1999; Schnee et al. 2008; Flagey et al. 2009; Planck Collaboration et al. 2011a). An example of a nearby galaxy which appears to harbor such large-scale variations is M 31 (Nieten et al. 2006). Evidence suggesting such variations is also observed in dwarf irregulars, particularly those with extended $\mathrm{H}$ i envelopes (Walter et al. 2007; Draine et al. 2007). In the case of the SMC, $\delta_{\mathrm{GDR}}$ appears to be systematically different in the Wing, the SW region of the Bar, and the NE region of the Bar (Leroy et al. 2011).

In the presence of systematic $\delta_{\mathrm{GDR}}$, variations adopting a single $\delta_{\mathrm{GDR}}$, although appealing in its simplicity, will lead to dramatically overpredicting the amount of $\mathrm{H}_{2}$ in regions that have an intrinsically lower $\delta_{\mathrm{GDR}}$. A more robust approach, and a considerably more conservative one, is to do a local average determination of $\delta_{\mathrm{GDR}}$, as a function of either position or some other environmental quantity like IR surface brightness. That is the procedure we follow, and the $\mathrm{H}_{2}$ results thus obtained are consistent with other observational constraints, such as virial masses on large scales (Leroy et al. 2009) and $\mathrm{H}_{2}$ column densities determined in absorption along diffuse lines of sight (Tumlinson et al. 2002). Nonetheless, $\delta_{\mathrm{GDR}}$ remains the largest source of systematic uncertainty in our estimation of $\Sigma_{\mathrm{H} 2}$. It is possible we are missing a large extended molecular component, although we consider it unlikely.
We implement this approach, making an iterative local measurement of $\delta_{\mathrm{GDR}}$. First, we blank regions of the $\tau_{160}$ and $\mathrm{H}$ I maps where bright $\mathrm{CO}$ is detected - these lines of sight are likely to include $\mathrm{H}_{2}$, so that $\Sigma_{\mathrm{H}_{\mathrm{I}}} / \tau_{160}$ is not a good tracer of the total $\delta_{\mathrm{GDR}}$. Next we smooth both maps with a kernel of radius $R \sim 200 \mathrm{pc}$ and calculate $\delta_{\mathrm{GDR}}=\Sigma_{\mathrm{HI}} / \tau_{160}$ for each region at this coarser resolution. This choice of kernel size represents a compromise between, on the one hand, calibrating $\delta_{\mathrm{GDR}}$ as close as possible to the molecular regions to minimize the effect of spatial variations and, on the other, avoiding including regions with significant $\mathrm{H}_{2}$ in its calibration. Using a kernel with $R \sim 450 \mathrm{pc}$ increases the total molecular mass of the SMC by $\sim 40 \%$, while reducing the kernel to $R \sim 100 \mathrm{pc}$ reduces the mass by $\sim 50 \%$, thus bracketing the impact of the choice of the smoothing scale. The $\delta_{\mathrm{GDR}}$ map produced this way has a median value $\delta_{\mathrm{GDR}} \approx 5.9 \times 10^{25} \mathrm{~cm}^{-2}$ and a spread of $\sim 0.4$ dex. Representative values for the N83/N84 region near the end of the Wing, the SW of the Bar, and the NE of the Bar are $\delta_{\mathrm{GDR}} \sim 5.9,4.8$, and $3.1 \times 10^{25} \mathrm{~cm}^{-2}$, respectively, with the highest values occurring near the central portions of the Wing. These values span the range of 8-14 times higher than Galactic, taking $\delta_{\mathrm{GDR}} \approx 4.1 \times 10^{24} \mathrm{~cm}^{-2}$ determined for Galactic cirrus as representative of the Milky Way (Boulanger et al. 1996).

We apply this low-resolution $\delta_{\mathrm{GDR}}$ to the nearby blanked regions with bright $\mathrm{CO}$ emission. We then apply Equation (1) to our full-resolution maps to estimate $\Sigma_{\mathrm{H} 2}$ everywhere. Because this may reveal new regions where $\mathrm{H}_{2}$ makes a significant contribution to the gas surface density we iterate the process once, blanking everywhere that has bright $\mathrm{CO}$ and everywhere with $\Sigma_{\mathrm{H} 2}>0.5 \Sigma_{\mathrm{HI}}$. We verified that iterating further does not significantly change the molecular surface densities. The result of this second iteration is our estimate for $\Sigma_{\mathrm{H} 2}$ at an angular resolution $\theta \sim 40^{\prime \prime}$ (Figure 1). The map shows very good correspondence with the recent optical reddening maps from Haschke et al. (2011).

We estimate the uncertainties in this map via a Monte Carlo calculation. In each iteration of this calculation, we add realistic noise to the data, adjust the zero point of the IR maps within the uncertainties, re-derive the relation used to predict $I_{70} / I_{160}$ from $I_{100} / I_{160}$, and propagate the noise in this relation forward. We also vary the wavelength dependence of the dust opacity, $\beta$, across its likely range $\beta \approx 1-2$, with one value randomly chosen for each iteration. We carry out 1000 such exercises and calculate the 1,2 , and $3 \sigma$ uncertainties in $T_{\text {dust }}$ and $\tau_{160}$ from the resulting distributions. These are propagated into uncertainties in $\Sigma_{\mathrm{H} 2}$. Using this technique we estimate our uncertainty in $\Sigma_{\mathrm{H} 2}$ before deprojection to be $1 \sigma \sim 15 M_{\odot} \mathrm{pc}^{-2}$ (equivalent to $\Sigma_{\mathrm{H} 2} \sim 11.5 M_{\odot} \mathrm{pc}^{-2}$ after correcting for the inclination angle of the SMC).

The following points are important to keep in mind, since they represent limitations of our $\mathrm{H}_{2}$ map and ultimately of our analysis. First, to remove spurious $\mathrm{H}_{2}$ emission from our map we only retain contiguous regions of area $\geqslant 4 \operatorname{arcmin}^{2}$ (this cleans up a few islets of emission) and $\Sigma_{\mathrm{H} 2}>23 M_{\odot} \mathrm{pc}^{-2}$ ( $2 \sigma$ deprojected), and set the rest of the map to $\Sigma_{\mathrm{H} 2}=0$. Because of this and the method used to derive a local $\delta_{\mathrm{GDR}}$, we cannot recover a pervasive $\mathrm{H}_{2}$ component. Other observations suggest that a large molecular component of this form is very unlikely (Dickey et al. 2000; Tumlinson et al. 2002); thus we do not view this as a significant uncertainty although it remains a possibility.

Second, the SMC has a complex geometry (see discussion in Section 1.1). As a result certain lines of sight may contain 

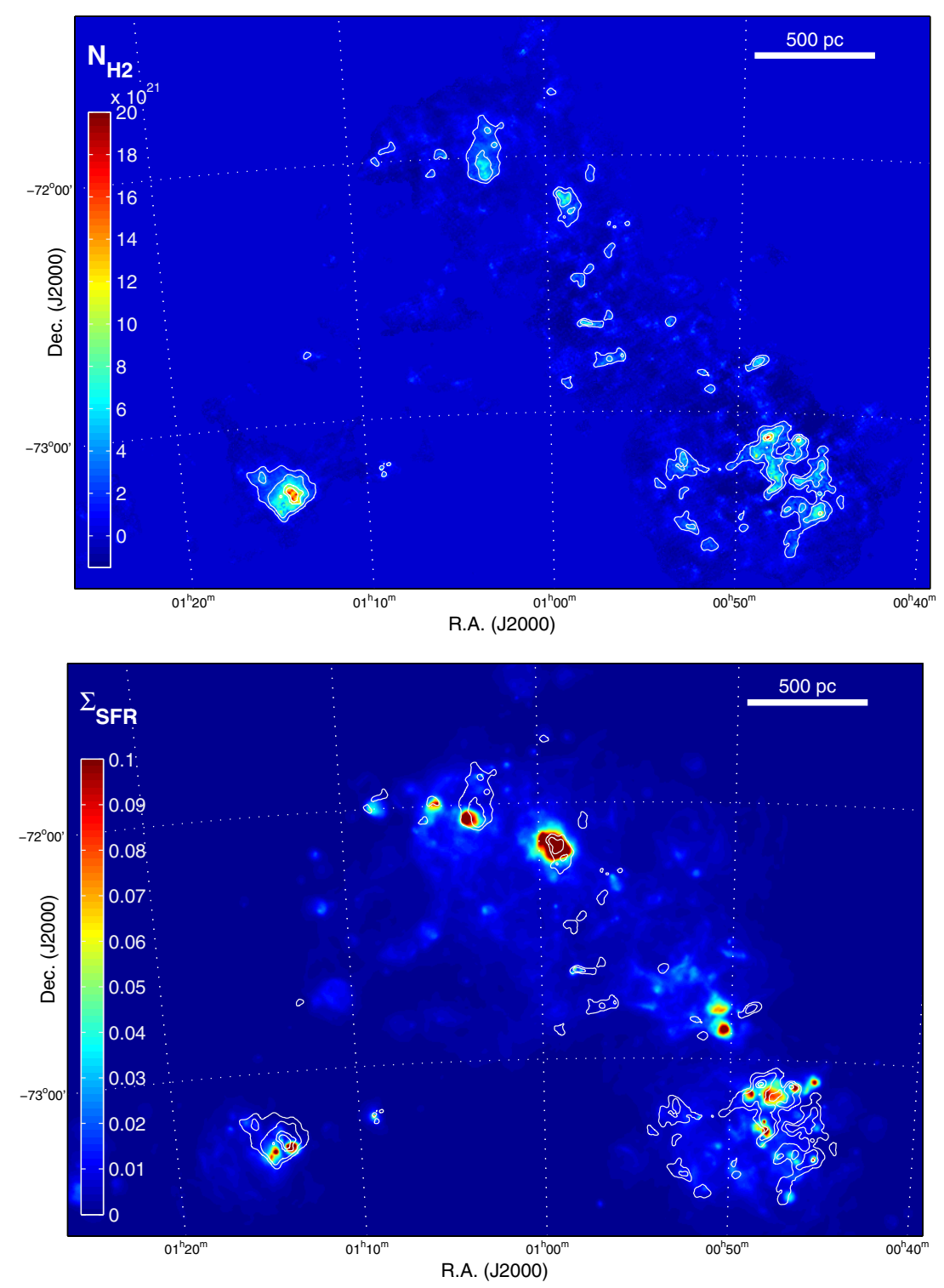

Figure 1. Top: $\mathrm{H}_{2}$ column density map at $\sim 12$ pe resolution. This map is obtained from modeling the Spitzer dust continuum observations from $\mathrm{S}^{3} \mathrm{MC} / \mathrm{SAGE}$-SMC (Bolatto et al. 2007; Gordon et al. 2011) together with the combined ATCA/Parkes $21 \mathrm{~cm} \mathrm{H} \mathrm{I} \mathrm{map} \mathrm{(Stanimirović} \mathrm{et} \mathrm{al.} \mathrm{1999).} \mathrm{The} \mathrm{color} \mathrm{bar} \mathrm{inset} \mathrm{indicates} \mathrm{the} \mathrm{values}$ for the color scale, in units of $10^{21} \mathrm{~cm}^{-2}$. The $N_{\mathrm{H} 2}$ contours are placed at $N_{\mathrm{H} 2} \approx 1.4,3.4,8$, and $12 \times 10^{21} \mathrm{~cm}^{-2}$, equivalent to deprojected molecular surface densities $\Sigma_{\text {mol }} \approx 23,56,130$, and $200 M_{\odot} \mathrm{pc}^{-2}$ when including the correction for the cosmic abundance of helium and the $40^{\circ}$ inclination of the source. The western region, oriented roughly SW to NE and harboring most of the star formation activity as well as the molecular gas, is called the SMC Bar. The extension to the SE is called the SMC Wing and is unremarkable in molecular gas except for the N83/N84 molecular cloud complex, which is the $\Sigma_{\mathrm{H} 2}$ peak of the galaxy. Bottom: $\mathrm{H}_{2}$ map overlaid on the unobscured $\Sigma_{\mathrm{SFR}}$ map derived from $\mathrm{H} \alpha$. The SFR is computed using the first term of Equation (4), and the color scale ranges from $\Sigma_{\mathrm{SFR}}=0$ to $0.1 M_{\odot} \mathrm{yr}^{-1} \mathrm{kpc}^{-2}$. There is good correlation between regions with star formation and regions with molecular gas, but not a one-to-one correspondence at high spatial resolution. The overall correlation improves when smoothing to scales of $200 \mathrm{pc}$ and larger. There is also a pervasive component of diffuse, low level H $\alpha$ emission.

a combination of regions with different $\delta_{\mathrm{GDR}}$. The presence of diffuse, high- $\delta_{\mathrm{GDR}}$ emission along the line of sight will invalidate Equation (1). The easiest way to correct for this is to subtract a "dust-free" component from the H I map to adjust the zero point of the dust-gas correlation. The likely magnitude of this dust-free $\mathrm{H}_{\mathrm{I}}$ component for the SMC is $\Sigma_{\mathrm{H}_{\mathrm{I}}} \sim 20-40 M_{\odot} \mathrm{pc}^{-2}$ (Leroy et al. 2011). We do not remove a "dust-free" component in the analysis presented here. Removing it would drive our $\mathrm{H}_{2}$ map toward lower values of $\Sigma_{\mathrm{H} 2}$.

Third, despite our attempt at minimizing the effect of systematic $\delta_{\mathrm{GDR}}$ changes through a local determination, variations in the dust emissivity and $\delta_{\mathrm{GDR}}$ between the dense and diffuse ISM are possible and largely unconstrained. If they have the expected sense of higher optical depths per unit gas in $\mathrm{H}_{2}$ than in
$\mathrm{H}$, they will drive the $\Sigma_{\mathrm{H} 2}$ toward values that are too high. Thus, the errors introduced have the same sign as those discussed in the previous point. Our best estimate of their combined effect is a factor-of-two systematic uncertainty in $\Sigma_{\mathrm{H} 2}$, with lower $\Sigma_{\mathrm{H} 2}$ values more likely.

Fourth, at $98^{\prime \prime}$ the resolution of the $\mathrm{H}$ I map is somewhat lower than that of the IR maps. Therefore, at spatial resolutions better than $r \sim 30 \mathrm{pc}$ we have assumed that $\Sigma_{\mathrm{H}_{\mathrm{I}}}$ is smooth at scales below the 1'.6 resolution of our H I map (Stanimirović et al. 1999). This assumption is almost certainly flawed in detail, but will average out across the whole galaxy, and it has no impact on quantities determined on $r \gtrsim 30$ pc scales.

Our estimate of a factor-of-two systematic uncertainty in our $\mathrm{H}_{2}$ determination, together with source geometry uncertainties 
discussed in Sections 1.1 and 3.3, results in a combined systematic uncertainty of $\sim 0.5$ dex (a factor of three) in the molecular depletion time in the SMC (Section 3.2).

\subsection{Estimating $\mathrm{H}_{2}$ from $\mathrm{CO}$}

The standard practice is to estimate the amount of molecular gas present in a system using ${ }^{12} \mathrm{CO}$ observations. This requires the use of a proportionality factor to convert intensity into column density or mass. We use the following equations:

$$
\begin{aligned}
& N\left(\mathrm{H}_{2}\right)=X_{\mathrm{CO}} I_{\mathrm{CO}} \\
& M_{\mathrm{mol}}=\alpha_{\mathrm{CO}} L_{\mathrm{CO}},
\end{aligned}
$$

where our adopted proportionality constants appropriate for Galactic gas are $X_{\mathrm{CO}}=2 \times 10^{20} \mathrm{~cm}^{-2}\left(\mathrm{~K} \mathrm{~km} \mathrm{~s}^{-1}\right)^{-1}$ and $\alpha_{\mathrm{CO}}=4.4 M_{\odot}\left(\mathrm{K} \mathrm{km} \mathrm{s}^{-1} \mathrm{pc}^{2}\right)^{-1}, I_{\mathrm{CO}}$ is the integrated intensity of the ${ }^{12} \mathrm{CO} J=1 \rightarrow 0$ transition (usually in $\mathrm{K} \mathrm{km} \mathrm{s}^{-1}$ ), and $L_{\mathrm{CO}}$ is the luminosity of the same transition (in $\mathrm{K} \mathrm{km} \mathrm{s}^{-1} \mathrm{pc}^{2}$ ). The resulting column density and mass are in $\mathrm{cm}^{-2}$ and $M_{\odot}$, respectively, and the molecular mass $M_{\text {mol }}$ corresponds to the mass of $\mathrm{H}_{2}$ corrected by the contribution of the cosmic abundance of He. Note that although the Galactic values are approximately appropriate on the small spatial scales in the CO-bright material (Bolatto et al. 2008), they are most likely inappropriate on the large scales (Rubio et al. 1993; Israel 1997; Israel et al. 2003; Leroy et al. 2007a, 2011). This is not surprising: if $\mathrm{CO}$ and $\mathrm{H}_{2}$ are not perfectly co-located their ratio will depend on the regions over which we are averaging. In particular, if $\mathrm{CO}$ is confined to the highly shielded high surface density cores while $\mathrm{H}_{2}$ is more widespread (e.g., Lequeux et al. 1994; Bolatto et al. 1999), $X_{\mathrm{CO}}$ will be small on CO-bright regions and large when measured on large spatial scales (CO freezeout into grains is not important on the spatial scales considered). We discuss this further in Section 3.

\subsection{Tracing Recent Star Formation}

We use $\mathrm{H} \alpha$ emission, locally corrected for extinction, to trace the surface density of recent star formation. The $\mathrm{H} \alpha$ observations we use were obtained, calibrated, continuum subtracted, and mosaicked by the Magellanic Cloud Emission Line Survey (MCELS; Smith \& The MCELS Team 1999). Particularly for bright stars, the continuum subtraction may leave noticeable artifacts, which we have masked out in our analysis. We convolve these images with the point-spread function of the MIPS $160 \mu \mathrm{m}$ camera, to match them to the $\sim 40^{\prime \prime}$ resolution of our molecular gas map.

We correct the ionizing photon flux inferred from $\mathrm{H} \alpha$ for the effects of extinction using the MIPS $24 \mu \mathrm{m}$ image obtained by SMC-SAGE, and the prescription from Calzetti et al. (2007). The implied extinctions are very small over most of the SMC, only becoming significant for the centers of the brightest $\mathrm{H}$ II regions. This is consistent with the findings of Caplan et al. (1996), who used the Balmer decrement technique to obtain a typical extinction $A_{\mathrm{H} \alpha} \sim 0.3$ mag toward bright SMC H II regions (see also Harris \& Zaritsky 2004 for a discussion of extinction based on fitting color-magnitude diagrams). The small extinction correction should not be surprising since the SMC is notoriously dust poor, with a dust-to-gas ratio that is a factor of 5-10 lower than Galactic (Leroy et al. 2007a). This galaxy simply does not harbor much obscured star formation activity.
The combination of $\mathrm{H} \alpha$ and $24 \mu \mathrm{m}$ maps yields an estimate of the extinction-corrected ionizing photon surface density. For our analysis we convert this to an SFR surface density, $\Sigma_{\mathrm{SFR}}$, following (Calzetti et al. 2007, Equation (7))

$$
\Sigma_{\mathrm{SFR}}=5.3 \times 10^{-42}\left[\Sigma_{\mathrm{H} \alpha}+0.031 \Sigma_{24}\right],
$$

where $\Sigma_{\mathrm{H} \alpha}$ and $\Sigma_{24}$ are, respectively, the surface densities of $\mathrm{H} \alpha$ and $24 \mu \mathrm{m}$ far-infrared emission, in erg $\mathrm{s}^{-1} \mathrm{kpc}^{-2}$, and the relation to SFR assumes an underlying broken powerlaw Kroupa initial mass function. This prescription is local, obtained for individual star-forming regions and calibrated against Paschen $\alpha$ emission. The use of $24 \mu \mathrm{m}$ emission to correct $\mathrm{H} \alpha$ has been tested in M 33 by Relaño et al. (2010) on the scale of individual H II regions. Calzetti et al. (2007) found the metallicity dependence of the extinction correction to be $\lesssim 20 \%$ for galaxies down to nebular metallicities below that of the SMC.

The very high spatial resolution of our data, $r \sim 12 \mathrm{pc}$ (though much longer along the line of sight) adds some complication to the concept of an SFR. The calibration by Calzetti et al. (2007) is derived on spatial scales of hundreds of parsecs. On the small scales corresponding to our full resolution any individual line of sight may poorly sample the high mass end of the initial mass function, be populated by stars resulting from a single star formation episode, or emit $\mathrm{H} \alpha$ radiation resulting from ionizing photons originating in an adjacent region. Spatial smoothing to larger scales removes these concerns. Throughout this paper we consider spatial scales ranging from $r \sim 12 \mathrm{pc}$ to the whole SMC. At the smallest of these scales, the idea of local SFR may break down, but at resolutions of $200 \mathrm{pc}$ or $1 \mathrm{kpc}$ (and certainly for the whole SMC) the application of a standard $\mathrm{H} \alpha$ to SFR conversion should be adequate. These scales also allow a fairer comparison to most other extragalactic studies.

It is important to note that in the results presented in the following sections we do not remove a diffuse ionized gas component, which may be as high as $40 \%$ of the total $\mathrm{H} \alpha$ emission in the SMC (Kennicutt et al. 1995). The 200-600 pc scales on which Calzetti et al. (2007) performed their calibration are much larger than the $\sim 12$ pc on which we carry out our study. Thus, it seems likely that part of the diffuse ionized emission due to the escape of ionizing photons from $\mathrm{H}$ II regions is already included in the calibration, suggesting that $40 \%$ is an upper bound to the SFR correction due to diffuse emission. To assess the impact of the diffuse $\mathrm{H} \alpha$ on our results we performed an analysis where we spatially filter the $\mathrm{H} \alpha$ image on several scales to remove the diffuse component, along the lines described in Rahman et al. (2011 and references therein). We found that the results presented in the following sections are robust to the presence of diffuse $\mathrm{H} \alpha$. The main effect of removing a diffuse ionized component is to correspondingly lengthen the gas depletion time, or equivalently somewhat lower the local star formation efficiency (SFE).

\section{RESULTS AND DISCUSSION}

Maps of $\Sigma_{\mathrm{H} 2}, \Sigma_{\text {gas }}$, and $\Sigma_{\mathrm{SFR}}$ allow us to study several aspects of the star formation law. In the following sections we will characterize the global properties of the SMC, compare molecular gas and star formation (the "molecular star formation law"), total neutral gas and star formation (the "total gas star formation law"), and measure the molecular fraction as a function of surface density. 


\subsection{Global Properties}

Our study allows us to derive a number of interesting integrated properties for the SMC. Integrating our extinctioncorrected $\mathrm{H} \alpha$ map, we obtain a global star formation rate $\mathrm{SFR}_{\mathrm{SMC}} \sim 0.037 M_{\odot} \mathrm{yr}^{-1}$. The extinction correction accounts for $\sim 10 \%$ of the global SFR. About $30 \%$ of the integrated $\mathrm{H} \alpha$ emission arises from extended low surface brightness regions, with equivalent SFR densities of $\Sigma_{\mathrm{H} \alpha}<5 \times 10^{-3} M_{\odot}$ $\mathrm{yr}^{-1} \mathrm{kpc}^{-2}$, likely reflecting long mean free paths for ionizing photons escaping $\mathrm{H}$ II regions (with perhaps some contribution from other sources of ionization). The global SFR we measure is very comparable to the present SFR obtained from the photometric analysis of the resolved stellar populations by Harris \& Zaritsky (2004), and only slightly lower than the $\mathrm{SFR}_{\mathrm{SMC}} \sim 0.05 M_{\odot} \mathrm{yr}^{-1}$ obtained by Wilke et al. (2004) based on the study of the SMC far-infrared emission. Note, however, that in the latter case the estimate is based on correcting up considerably the standard FIR calibration by assuming a much smaller dust optical depth in the SMC. It also relies on the synthetic model grid of Leitherer \& Heckman (1995), which has been superseded by the newer models incorporated in the Calzetti et al. (2007) calibration. In any case, this range of values is representative of the typical uncertainties in determining SFRs, also applicable to our value.

Integrating the molecular mass over the regions of the map with signal above our $2 \sigma$ threshold of $23 M_{\odot} \mathrm{pc}^{-2}$, we obtain $M_{\mathrm{SMC}}^{\mathrm{mol}} \sim 2.2 \times 10^{7} M_{\odot}$. This is an $\sim 35 \%$ revision down from previous studies using the same technique (Leroy et al. 2007a), mostly due to differences in the estimation of the dust-to-gas ratio and well within the factor of $\sim 2$ systematic uncertainties present in the analysis (for discussions see Leroy et al. 2007a, 2009). The global CO luminosity of the SMC is approximately $1 \times 10^{5} \mathrm{~K} \mathrm{~km} \mathrm{~s}^{-1} \mathrm{pc}^{2}$ (Mizuno et al. 2001; assuming a 20\% correction for flux in unmapped regions). This yields a globally averaged conversion factor from $\mathrm{CO}$ to molecular mass of $\alpha_{\mathrm{CO}} \sim 220 M_{\odot}\left(\mathrm{K} \mathrm{km} \mathrm{s}^{-1} \mathrm{pc}^{2}\right)^{-1}$. We return to $\alpha_{\mathrm{CO}}$ in the next section.

The gas depletion time, $\tau_{\mathrm{dep}}$, is a convenient way to parameterize the normalized SFR: $\tau_{\text {dep }}$ is the time needed for the present SFR to exhaust the existing gas reservoir (Young et al. 1986). Sometimes the inverse of this quantity is presented as a so-called SFE, indicating the fraction of the gas reservoir used in $10^{8} \mathrm{yr}$. Galaxies with high SFE can only sustain their present star formation activity for a short period of time, and thus are experiencing a starburst.

From this integrated SFR and $M_{\mathrm{mol}}$, the global molecular gas depletion time of the SMC is approximately $\tau_{\mathrm{dep}}^{\mathrm{mol}}=$ $M_{\text {mol }} /$ SFR $\sim 0.6$ Gyr. For comparison, the global molecular depletion time inferred from the $\mathrm{CO}$ luminosity and a Galactic conversion factor $\left(\alpha_{\mathrm{CO}}=4.4\right)$ would be $\sim 0.01 \mathrm{Gyr}$. Although a Galactic conversion factor is clearly inappropriate, this exercise highlights the need for an alternative tracer of $\mathrm{H}_{2}$. The $\mathrm{CO}$ in the SMC is either dramatically underluminous for the observed level of star formation activity, or the SMC is in the midst of a starburst that will exhaust the molecular gas reservoir in only $\sim 10^{7} \mathrm{yr}$. Although the study of the global star formation history of the SMC by Harris \& Zaritsky (2004) finds its present SFR to be somewhat larger than the past average, the magnitude of that effect cannot explain such an implausibly short molecular gas depletion time.

Given its atomic mass $\left(M_{\mathrm{HI}} \simeq 4.2 \times 10^{8} M_{\odot}\right.$; Stanimirović et al. 1999), the SMC keeps only $~ 5 \%$ of its gas in the molecular phase. Thus, it appears to be genuinely poor at forming GMCs.

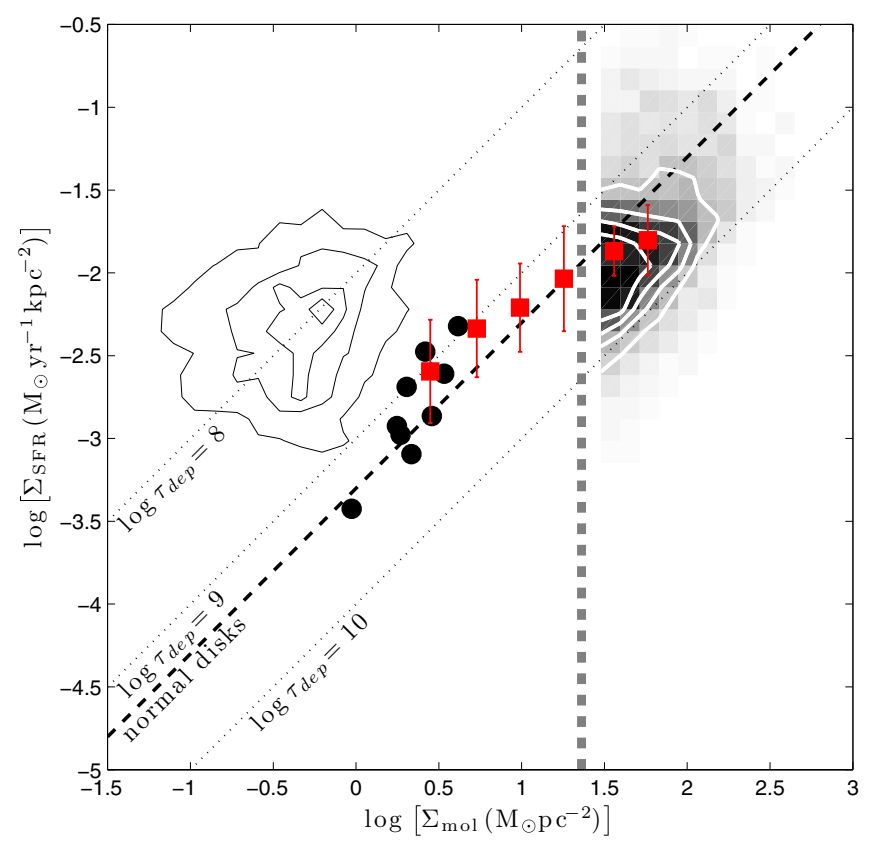

Figure 2. Molecular star formation law in the SMC. The gray scale shows the binned two-dimensional distribution of the $\Sigma_{\mathrm{SFR}}$ to $\Sigma_{\mathrm{mol}}$ correlation at a resolution $r \sim 12 \mathrm{pc}$, where $\Sigma_{\mathrm{mol}}$ is derived from the dust modeling. The intensity scale is proportional to the number of points that fall in a bin, with white contours indicating levels that are $20 \%, 40 \%, 60 \%$, and $80 \%$ of the maximum. The vertical gray dashed line indicates our adopted $2 \sigma$ sensitivity cut for the $r \sim 12 \mathrm{pc}$ data. The red squares and black circles show the results after spatial smoothing to $r \sim 200 \mathrm{pc}$ and $r \sim 1 \mathrm{kpc}$, respectively (the sensitivity limit has been moved down accordingly, assuming Gaussian statistics). The bars in the $r \sim 200 \mathrm{pc}$ data show the standard deviation after averaging in $\Sigma_{\mathrm{mol}}$ bins. The black contours, placed at the same levels as the white contours, show the distribution of $\Sigma_{\text {mol }}$ derived from CO observations (Mizuno et al. 2001) using the Galactic CO-to- $\mathrm{H}_{2}$ conversion factor. The dotted lines indicate constant molecular gas depletion times $\tau_{\text {dep }}^{\mathrm{mol}}=0.1,1$, and $10 \mathrm{Gyr}$. The dashed line indicates the typical depletion time for normal disk galaxies $\tau_{\mathrm{dep}}^{\mathrm{mol}} \sim 2 \times 10^{9} \mathrm{yr}$ (Bigiel et al. 2008). The $\tau_{\text {dep }}^{\text {mol }}$ in the SMC is consistent with the range observed in normal disks for $\Sigma_{\text {mol }}$ derived from dust modeling.

(A color version of this figure is available in the online journal.)

The corresponding total gas depletion time is $\tau_{\text {dep }}^{\text {gas }} \sim 11.8 \mathrm{Gyr}$, so the SMC has enough fuel to sustain its current rate of star formation for approximately a Hubble time. By comparison, the median total gas depletion time for the 12 large galaxies in the THINGS/HERACLES sample is $\tau_{\text {dep }}^{\text {gas }} \approx 6.0$ Gyr (Leroy et al. 2008). Although a wide range of depletion times are present, $1<\tau_{\text {dep }}^{\text {gas }}<14$ Gyr, 10 of these galaxies have $\tau_{\text {dep }}^{\text {gas }}<8$ Gyr. In contrast, in H I-dominated dwarf galaxies and the outer disks of spirals $\tau_{\text {dep }}^{\text {gas }} \gtrsim 10$ Gyr (Bigiel et al. 2010b).

\subsection{Relation between Molecular Gas and Star Formation}

Figure 2 shows the correlation between molecular gas and star formation activity traced by the extinction-corrected $\mathrm{H} \alpha$ at several spatial resolutions: $r \sim 12$ pc (gray scale), $r \sim 200 \mathrm{pc}$ (red squares, binned), and $r \sim 1 \mathrm{kpc}$ (black circles). The gray area and the corresponding white contours show that a fairly well-defined relation exists between the star formation activity and the surface density of molecular gas obtained from the dust continuum modeling described in Section 2.1 even on our smallest scales.

Note that the $x$ and $y$ axes of this plot are not implicitly correlated. The abscissa contains information from the 70 , 100 , and $160 \mu \mathrm{m}$ far-infrared continuum as well as the $\mathrm{HI}_{\mathrm{I}}$ 
map, while the ordinate is chiefly $\mathrm{H} \alpha$. The small extinction correction derived from the $24 \mu \mathrm{m}$ data has a negligible effect on the correlation. Also note that because we correct for dust temperature when deriving the dust surface density, $\Sigma_{\mathrm{mol}}$ should, in principle, also be independent of heating effects.

The molecular gas depletion time depends on the scale considered (Figure 2). On the smallest scales considered, $r \sim 12 \mathrm{pc}$, the depletion time in the molecular gas is $\log \left[\tau_{\mathrm{dep}}^{\mathrm{mol}} /(1 \mathrm{Gyr})\right] \sim$ $0.9 \pm 0.6\left(\tau_{\mathrm{dep}}^{\mathrm{mol}} \sim 7.5 \mathrm{Gyr}\right.$ with a factor of 3.5 uncertainty after accounting for observed scatter and systematics involved in producing the $\mathrm{H}_{2}$ map as well as the geometry of the source). As mentioned in the previous section, $\tau_{\mathrm{dep}}^{\mathrm{mol}}$ shortens when considering larger spatial scales due to the fact that the $\mathrm{H} \alpha$ and $\mathrm{H}_{2}$ distributions differ in detail, but are well correlated on scales of hundreds of parsecs (Figure 1). On size scales of $r \sim 200 \mathrm{pc}$ (red squares in Figure 2), corresponding to very good resolution for most studies of galaxies beyond the Local Group, the molecular depletion time is $\log \left[\tau_{\mathrm{dep}}^{\mathrm{mol}} /(1 \mathrm{Gyr})\right] \sim 0.2 \pm 0.3$, or $\tau_{\text {dep }}^{\text {mol }} \approx 1.6$ Gyr. The depletion time on $r \sim 1 \mathrm{kpc}$ scales (black circles in Figure 2), corresponding to the typical resolution of extragalactic studies, stays constant for the central regions of our map (where the smoothing can be accurately performed), $\log \left[\tau_{\mathrm{dep}}^{\mathrm{mol}} /(1 \mathrm{Gyr})\right] \sim 0.2 \pm 0.2$. Thus, our results converge on the scales typically probed by extragalactic studies. This constancy reflects the spatial scales over which $\mathrm{H} \alpha$ and molecular gas are well correlated. Although the precise values differ, a very similar trend for $\tau_{\mathrm{dep}}^{\mathrm{mol}}$ as a function of spatial scale is observed in M 33 (Schruba et al. 2010). The further reduction of the depletion time when considering the entire galaxy $\left(\tau_{\mathrm{dep}}^{\mathrm{mol}} \approx 0.6 \mathrm{Gyr}\right)$ reflects the contribution from a component of extended $\mathrm{H} \alpha$ emission, which is filtered out in the calibration of the SFR indicator (Calzetti et al. 2007; Rahman et al. 2011). The SMC is on the high end of the observed distribution of values for the fraction of diffuse $\mathrm{H} \alpha$, but fractions of $40 \%-50 \%$ are common in galaxies (e.g., Hoopes et al. 1999).

Within the uncertainties, our results are not significantly different from the mean $\mathrm{H}_{2}$ depletion time obtained in studies of molecule-rich late-type disks on $750 \mathrm{pc}$ to $1 \mathrm{kpc}$ spatial scales, where $\tau_{\text {dep }}^{\mathrm{mol}} \sim 2.0 \pm 0.8 \mathrm{Gyr}$ averaged over regions with molecular emission (SFE $\approx 5 \%$ within $0.1 \mathrm{Gyr}$; Bigiel et al. 2008, 2011; Leroy et al. 2008). This is the methodology used in resolved studies of $\tau_{\mathrm{dep}}^{\mathrm{mol}}$ in more distant galaxies, and our results are directly comparable.

This contrasts sharply with the results using the $\mathrm{CO}$ map (black contours) obtained by the NANTEN telescope (Mizuno et al. 2001), using a Galactic CO-to- $\mathrm{H}_{2}$ conversion (the assumption in many extragalactic studies). The $\mathrm{CO}$ distribution is offset by a factor of $\sim 40$ from the $\mathrm{H}_{2}$ distribution. This offset corresponds to the most common $\alpha_{\mathrm{CO}}$ implied by our dust-map, $\alpha_{\mathrm{CO}} \approx 185 M_{\odot}\left(\mathrm{K} \mathrm{km} \mathrm{s}^{-1} \mathrm{pc}^{2}\right)^{-1}$ (very similar to the global $\alpha_{\mathrm{CO}}$ ). Both values of $\alpha_{\mathrm{CO}}$ are broadly consistent with CO-to- $\mathrm{H}_{2}$ conversion factors obtained by previous dust continuum modeling and virial mass techniques on large scales (Rubio et al. 1993; Israel 1997; Leroy et al. 2007a, 2011), though factors of two to three discrepancy persist from study to study. They differ, however, from estimates based on small-scale virial masses toward the CO-bright peaks, which tend to obtain values of $\alpha_{\mathrm{CO}}$ closer to Galactic (Israel et al. 2003; Blitz et al. 2007; Bolatto et al. 2008; Müller et al. 2010). This discrepancy between COto- $\mathrm{H}_{2}$ conversion factor on the large and on the small scales can be understood in terms of the existence and mass dominance of large molecular envelopes poor in CO. Such envelopes are ex-

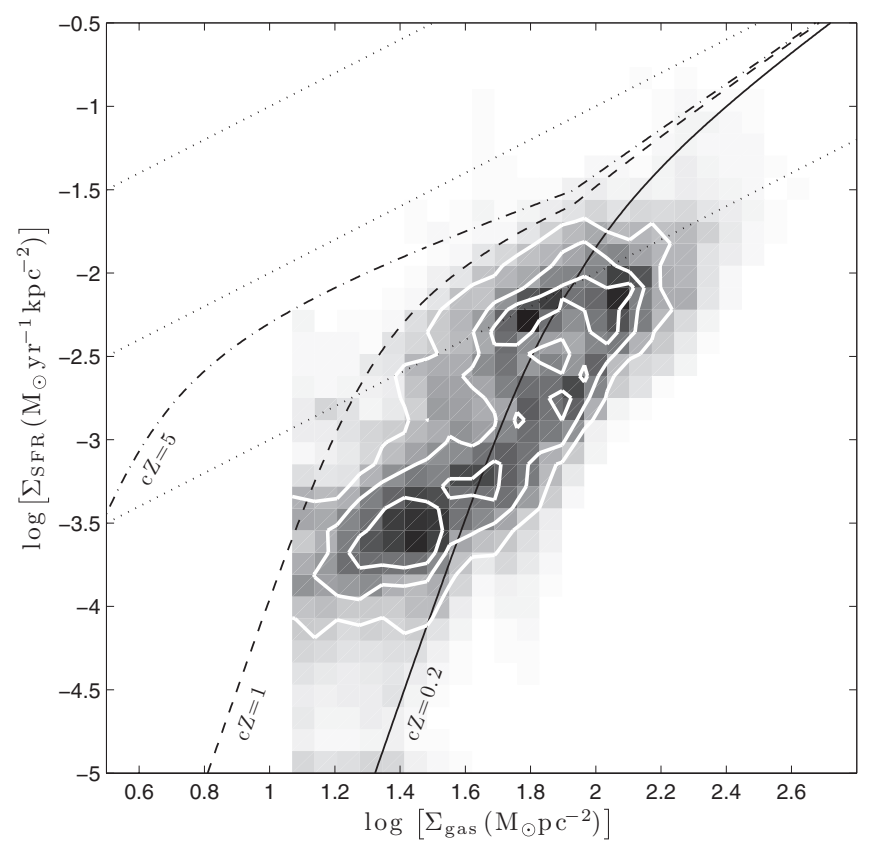

Figure 3. Total gas star formation law in the SMC. The gray scale shows the two-dimensional distribution of the correlation between $\Sigma_{\text {SFR }}$ and $\Sigma_{\text {gas }}$, where $\Sigma_{\text {gas }}$ is the surface density of atomic plus molecular gas corrected by helium. The white contours indicate the correlation due to atomic gas alone, which dominates the gas mass (and $\Sigma_{\text {gas }}$ ) in the SMC. The contour levels, and the dotted lines indicating constant $\tau_{\mathrm{dep}}^{\mathrm{gas}}$, are at the same values as in Figure 2. The dash-dotted, dashed, and solid lines indicate the loci of the model by Krumholz et al. (2009c, KMT09) for clumping factor by metallicity products $c Z=5,1$, and 0.2 , respectively. The first two bracket the behavior of most galaxies observed at $750 \mathrm{pc}$ resolution (see KMT09, Figure 1), while $c Z=0.2$ would be the value expected for the SMC with unity clumping factor (a reasonable assumption for the spatial resolution of the observations presented here, $r \sim 12 \mathrm{pc}$ ). Note that the surface density at which H I starts to saturate in the SMC is $\Sigma_{\mathrm{H}_{\mathrm{I}}} \sim 50 M_{\odot} \mathrm{pc}^{-2}$ (the typical surface density is $\Sigma_{\mathrm{H}_{\mathrm{I}}} \sim 85 \mathrm{M}_{\odot} \mathrm{pc}^{-2}$ at the full resolution of the $\mathrm{H}$ I data), considerably larger than the typical value in normal metallicity galaxies where $\Sigma_{\mathrm{H}_{\mathrm{I}}} \lesssim 10 \mathrm{M}_{\odot} \mathrm{pc}^{-2}$ (Bigiel et al. 2008, 2011). As a consequence any use of a "standard" total-gas-star-formation correlation for the SMC would dramatically underpredict total gas surface densities, or overpredict star formation activity. This is not true for molecular gas, as we discuss in the previous figure.

pected at all metallicities (Glover \& Mac Low 2011), and at the low metallicity of the SMC they likely constitute the dominant reservoir of molecular gas (Wolfire et al. 2010).

\subsection{Relation between Total Gas and Star Formation}

Figure 3 shows the total gas star formation law for the SMC, the relation between $\Sigma_{\mathrm{SFR}}$ and total $\left(\mathrm{HI}+\mathrm{H}_{2}\right)$ gas surface density $\Sigma_{\text {gas }}$, as well as $\Sigma_{\mathrm{SFR}}$ versus $\Sigma_{\mathrm{HI}}$ (which is almost the same, as atomic gas dominates). This relationship may be more complex than the molecular star formation law, resulting from a combination of phase balance in the ISM and the relative efficiencies of different types of gas at forming stars. Using a power-law ordinary least-squares bisector fit we find that $\log \left(\Sigma_{\mathrm{SFR}}\right)=(2.2 \pm 0.1) \log \left(\Sigma_{\text {gas }}\right)+(-6.5 \pm 0.1)$ for $\Sigma_{\text {gas }}>10 M_{\odot} \mathrm{pc}^{-2}$ at the full spatial resolution of the observations. This is very similar to the typical $1+p \approx 2$ slope measured for this relation in $\mathrm{HI}$-dominated regions of galaxies (Bigiel et al. 2010b), or the typical power-law index of the $\Sigma_{\text {SFR }}$ to $\Sigma_{\mathrm{H}}$ relation observed in faint dwarfs by Roychowdhury et al. (2009).

Thus, the relation between $\Sigma_{\mathrm{SFR}}$ and $\Sigma_{\text {gas }}$ is steep in the SMC, and similar to $\Sigma_{\text {SFR }}$ versus $\Sigma_{\text {gas }}$ at low surface density 
in normal galaxies, but the relation in the SMC is noticeably displaced toward larger total gas surface densities compared to large spirals (this was already evident in the study by Kennicutt et al. 1995). The atomic surface density in normal metallicity galaxies almost never exceeds a saturation point of $\Sigma_{\mathrm{HI}} \approx 10 M_{\odot} \mathrm{pc}^{-2}$ (Bigiel et al. 2008) averaged over $\sim \mathrm{kpc}$ scales, although smaller-scale higher-column H I clouds are observed in the Milky Way (e.g., Heiles \& Troland 2003). That is not the case in the SMC, where $\Sigma_{\mathrm{HI}}$ reaches values of $\sim 100 M_{\odot} \mathrm{pc}^{-2}\left(N(\mathrm{HI}) \sim 1 \times 10^{22} \mathrm{~cm}^{-2}\right.$; see the white contours in Figure 3). This is not purely the result of the high spatial resolution; high $\Sigma_{\mathrm{H}_{\mathrm{I}}}$ persists even averaged over large spatial scales (Stanimirović et al. 1999). It may be partially enhanced by the complex geometry of the source; the SMC is an interacting galaxy that may have significant elongation along the line of sight, not a simple flat disk (e.g., Yoshizawa \& Noguchi 2003). All surface densities (averaged over large scales) would be reduced by a factor of $\cos i / 0.77$ if the geometry is disklike and inclination exceeds $40^{\circ}$, or possibly by a larger factor if the galaxy is also elongated along the line of sight. These corrections are not large enough to explain the full magnitude of the effect we see, in light of the Hi kinematic analysis by Stanimirović et al. (2004), but could conceivably contribute a factor of $\sim 1.5-2$ (where the $50 \%$ factor corresponds to changing the inclination from $i=40^{\circ}$ to $i=60^{\circ}$, and a further $20 \%-30 \%$ factor is an estimate for elongation, obtained by evaluating the contribution from gas at high velocities to $\Sigma_{\mathrm{H}_{\mathrm{I}}}$; see Section 3.4).

This displacement of appreciable star formation activity toward high gas surface densities means that applying the Kennicutt-Schmidt relation observed for normal galaxies to the total gas surface density in the SMC would lead to a dramatic overprediction of the SFR. For example, in a normal disk we would expect total gas surface densities of $\Sigma_{\text {gas }} \sim 20 M_{\odot} \mathrm{pc}^{-2}$ to be associated with SFRs of $\Sigma_{\mathrm{SFR}} \sim 0.01 M_{\odot} \mathrm{yr}^{-1} \mathrm{kpc}^{-2}$ on $\sim 1 \mathrm{kpc}$ scales (Bigiel et al. 2008, 2011), while in the SMC the typical SFR associated with such gas surface density is one to two orders of magnitude lower. Conversely, using the observed SFR to estimate gas content (as it is sometimes done in studies of the high redshift universe) would lead to dramatic underestimates of the amount of total gas present.

This offset in the total gas star formation law stands in stark contrast to the conclusions in Section 3.2 about the relation between star formation activity and molecular gas, where the SMC appeared very similar to normal disk galaxies. The fact that the molecular SFE in the SMC is within the range observed in other galaxies implies that, within the usual (factor of three) uncertainties, the observed SFR accurately reflects the amount of molecular gas present.

This difference implies that there are more quantitative similarities between the distributions of molecular gas and recent star formation than between atomic gas and recent star formation. We plot this directly in Figure 4, which compares the cumulative distributions of star formation and gas surface density at $\sim 200$ pc resolution, a few times larger than the size of a large GMC in the Milky Way. This represents a typical size scale over which gas and star formation should be correlated. The abscissa in Figure 4 corresponds to the fraction of the total SFR below a particular value of the $\Sigma_{\mathrm{SFR}}$. The ordinate corresponds to the fraction of gas accumulated in those lines of sight.

Figure 4 shows that star formation and molecular gas track linearly with each other, while the nonlinear shape of the $\Sigma_{\mathrm{H}_{\mathrm{I}}}$ distribution reflects the fact that most of the $\mathrm{H}$ I is found on lines

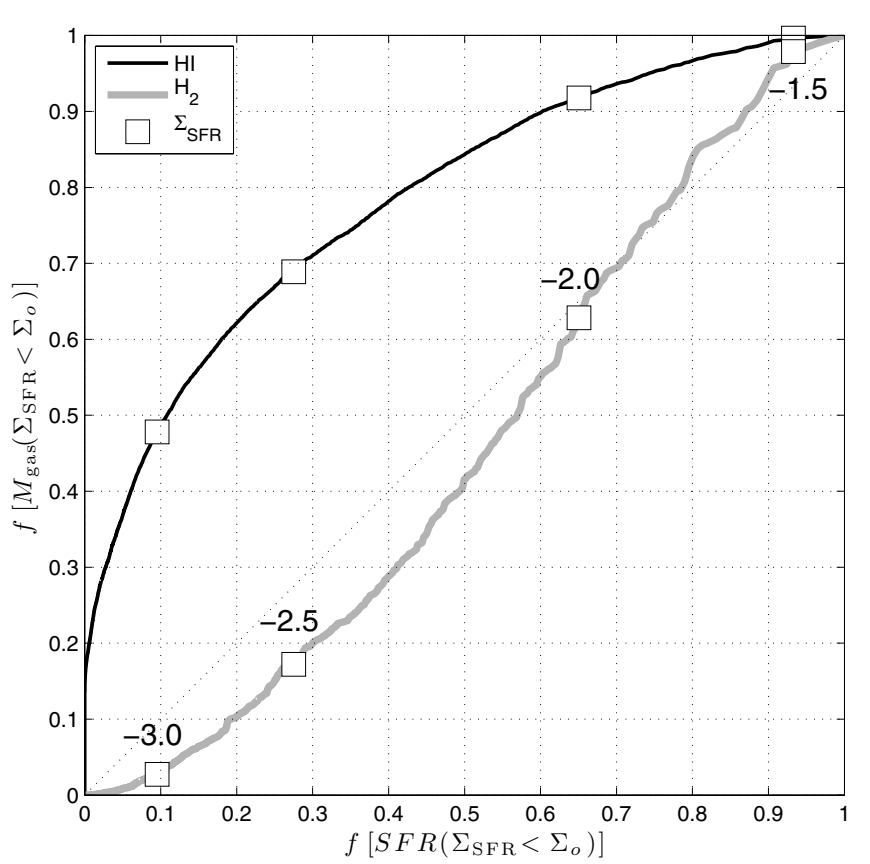

Figure 4. Cumulative distribution function of gas mass in lines of sight with increasing star formation activity at a spatial resolution of $200 \mathrm{pc}$, plotted against the cumulative distribution function of SFR for the same lines of sight. The abscissa corresponds to the fraction of the total SFR below a particular value of the $\Sigma_{\mathrm{SFR}}$. The ordinate corresponds to the fraction of gas ( $\mathrm{H}$ i in black, $\mathrm{H}_{2}$ in gray) accumulated in those lines of sight. The squares show the locations of particular values for $\log \left[\Sigma_{\mathrm{SFR}}\right]$, in units of $M_{\odot} \mathrm{yr}^{-1} \mathrm{kpc}^{-2}$. For example, about $28 \%$ of all the extinction-corrected $\mathrm{H} \alpha$ emission in the SMC is found in lines of sight with corresponding surface densities $\Sigma_{\mathrm{SFR}} \lesssim 10^{-2.5} M_{\odot} \mathrm{yr}^{-1} \mathrm{kpc}^{-2}$, and those lines of sight contain $\sim 69 \%$ and $\sim 17 \%$ of all the atomic and molecular mass, respectively. This plot highlights the fact that most $\mathrm{H}_{\mathrm{I}}$ is found in regions with little star formation, even on scales of $200 \mathrm{pc}$.

of sight with little star formation activity. A similar phenomenon is observed in other faint dwarf galaxies (Roychowdhury et al. 2009 , 2011). This lack of correspondence between H I and $\Sigma_{\text {SFR }}$ is even more acute at $r \sim 12 \mathrm{pc}$, while $\mathrm{H}_{2}$ and star formation continue to track each other well. Approximately $85 \%$ by mass of the $\mathrm{HI}_{\mathrm{I}}$ in the SMC is in the warm phase, according to observation and modeling of 28 lines of sight toward background continuum sources (Dickey et al. 2000). Together with Figure 4, this strongly suggests that most $\mathrm{HI}$ in the SMC is not directly related to the star-forming gas, i.e., it does not belong to atomic envelopes of molecular clouds.

\subsection{Molecular Gas Fraction}

Figure 5 shows our final basic observational result, the molecular fraction of the SMC as a function of total gas surface density. Gray scale shows the density of our data in $\left(\Sigma_{\mathrm{H} 2} / \Sigma_{\mathrm{HI}}\right)-\left(\Sigma_{\mathrm{gas}}\right)$ space at $200 \mathrm{pc}$ resolution; the blue contours show it at $12 \mathrm{pc}$ resolution. The curved labeled lines indicate model predictions by Krumholz et al. (2009c). At $\sim \mathrm{kpc}$ resolution, most massive star-forming disk galaxies lie between the curves labeled $c Z=1$ and $c Z=5$ (see Section 4.1). For a given total gas surface density, the SMC has molecular fractions much lower than these large galaxies with the offset often an order of magnitude or more. We discussed in Section 1.1 the fact that the complex distribution of $\mathrm{H}$ in the SMC along the line of sight is a source of uncertainty. We can obtain a rough estimate of the effects of H I not associated with the disk of the SMC on the molecular ratio by recalculating $\Sigma_{\mathrm{HI}_{\mathrm{I}}}$ after removing $\mathrm{H}_{\mathrm{I}}$ emission outside 


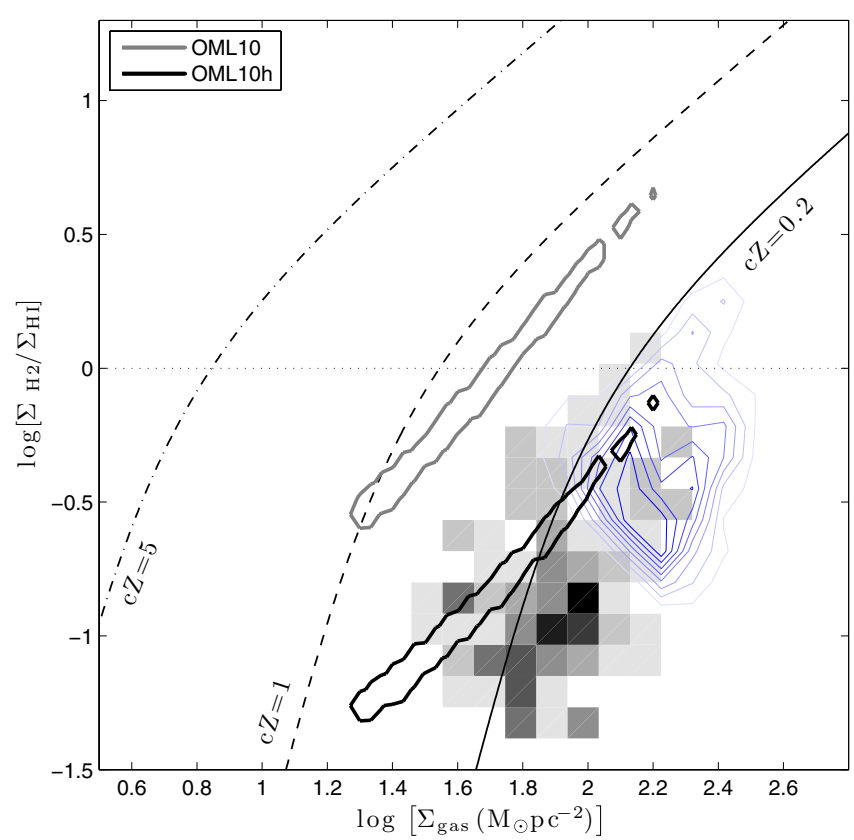

Figure 5. Ratio of molecular-to-atomic gas in the SMC. The blue contours and the gray scale show the two-dimensional distribution of the ratio $\Sigma_{\mathrm{H}_{2}} / \Sigma_{\mathrm{H}_{\mathrm{I}}}$ vs. $\Sigma_{\text {gas }}$ on scales of $r \sim 12 \mathrm{pc}$ and $r \sim 200 \mathrm{pc}$, respectively (note that the hard edge present in the blue contours at low ratios and low $\Sigma_{\text {gas }}$ is the result of our adopted $2 \sigma$ cut in $\Sigma_{\mathrm{H} 2}$ ). The dotted horizontal line indicates $\Sigma_{\mathrm{H} 2} / \Sigma_{\mathrm{H}_{\mathrm{I}}}=1$, denoting the transition between the regimes dominated by $\mathrm{H}_{\mathrm{I}}$ and $\mathrm{H}_{2}$. The dash-dotted, dashed, and solid lines show the predictions of KMT09 for different values of the $c Z$ parameter, as in Figure 3. For the SMC, $c Z=0.2$ at $r \sim 12 \mathrm{pc}$, and the KMT09 curve overestimates the molecular-to-atomic ratio by a factor of two to three. At $r \sim 200 \mathrm{pc}$ we may expect $c Z \approx 1$ using the standard clumping factor $c=5$ adopted by KMT09 for unresolved complexes. Although molecular gas in the SMC is highly clumped, the atomic gas is not, so the $c Z=1$ curve overestimates $\Sigma_{\mathrm{H} 2} / \Sigma_{\mathrm{H} \text { I }}$ at $200 \mathrm{pc}$. The thick gray and black contours indicate the predicted surface density ratio of gas in gravitationally bound complexes to diffuse gas, $\Sigma_{\mathrm{gbc}} / \Sigma_{\mathrm{diff}}$, in OML10 and in the model modified to incorporate the extra heating of in the diffuse gas (OML10h; see Section 4.2.1), respectively. The contours are calculated for the metallicity and distribution of stellar plus dark matter density in the SMC. The original OML10 prediction for $\Sigma_{\text {gbc }} / \Sigma_{\text {diff }}$ is considerably higher than the observed $\Sigma_{\mathrm{H} 2} / \Sigma_{\mathrm{HI}}$. The tightness of the contours is due to the fact that the self-gravity of the gas dominates over the stellar plus dark matter contribution, thus there is an almost one-to-one correspondence between $\Sigma_{\text {gas }}$ and the prediction for $\Sigma_{\text {gbc }} / \Sigma_{\text {diff }}$.

(A color version of this figure is available in the online journal.)

the velocity range $v_{\mathrm{lsr}} \approx 120-180 \mathrm{~km} \mathrm{~s}^{-1}$, taken to be representative of the disk of the galaxy. We find that this exercise lowers $\Sigma_{\mathrm{H}}$ by at most $30 \%$ the faint regions of the Wing, and more typically $\sim 10 \%-15 \%$. This is a small correction in the molecular ratio, well within our uncertainties in $\Sigma_{\mathrm{H} 2}$ alone, and although it may significantly contribute to the observed dispersion in $\Sigma_{\mathrm{H} 2} / \Sigma_{\mathrm{H}}$, it cannot be the cause of its offset with respect to normal galaxies. The SMC is strikingly bad at turning its wealth of atomic gas into molecular gas, particularly given the very high surface densities found in this galaxy.

\subsection{Synthesis of Results}

Using our dust-based $\Sigma_{\mathrm{H} 2}$ map, we showed that to first order the molecular star formation law in the SMC resembles that in disk galaxies. There is still room within the uncertainties for a factor-of-two-to-three decrease in $\tau_{\mathrm{dep}}^{\mathrm{mol}}$, but our best estimates at $0.2-1 \mathrm{kpc}$ resolution imply very good agreement between this low-metallicity dwarf and more massive disk galaxies. Note that since the scaling is linear, this result is insensitive to uncertainty in inclination or other aspects of the SMC's geometry. By contrast, the total gas star formation law is offset significantly from that observed in large galaxies. The SMC harbors unusually high $\Sigma_{\mathrm{HI}}$ and low $\Sigma_{\mathrm{SFR}}$ at a fixed total gas surface density (although the $\Sigma_{\text {SFR }}$ versus $\Sigma_{\text {gas }}$ distribution moves closer to the loci of large spirals if the star and gas are in a disk inclined by $i>40^{\circ}$, or if the galaxy is elongated along the line of site). At a given $\Sigma_{\text {gas }}$, the molecular gas fraction is also offset to values lower than those observed in massive disk galaxies, by typically an order of magnitude.

Two natural corollaries emerge from these observations. First, molecular clouds in the SMC are not extraordinarily efficient at turning gas into stars; star formation proceeds in them at a pace similar to that in GMCs belonging to normal disk galaxies. This suggests that, down to at least the metallicity of the SMC $\left(Z \sim Z_{\odot} / 5\right)$, the lower abundance of heavy elements does not have a dramatic impact on the microphysics of the star formation process, although it does appear to have an important effect at determining the fraction of the ISM capable of forming stars.

This is not a foregone conclusion. For example, it is conceivable that the low abundance of carbon and the consequent low dust-to-gas ratio and low extinction would affect the ionization fraction in the molecular gas. This may result in changes in the coupling with the magnetic field, perhaps slowing the GMC collapse and resulting in lower SFE and longer $\tau_{\text {dep }}^{\text {mol }}$. Or, alternatively, the low abundance of CO (an important gas coolant in dense molecular cores) could make it difficult for cores to shed the energy of gravitational contraction, slowing their collapse and again resulting in longer timescales for consuming the molecular gas (but see Krumholz et al. 2011). Our result implies that to first order metallicity does not have a large impact on the rate at which star formation proceeds locally in molecular gas in the SMC. Firming up this conclusion, however, requires detailed studies of molecular cloud lifetimes (for example, see Fukui et al. 1999).

Second, these observations provide very strong evidence that star formation activity is related directly to the amount molecular gas, with H I coupled to SFR only indirectly. This should be tempered by the consideration that, as pointed out in Section 2.1, our dust-derived $\mathrm{H}_{2}$ map may include a contribution from very cold, strongly self-absorbed $\mathrm{H}$ I (such as that sometimes associated with molecular cloud envelopes), which we cannot easily disentangle from molecular material in our analysis. The strong relation between molecular material and star formation explains some puzzling observations in the context of $\mathrm{HI}$ dominated systems. Wolfe \& Chen (2006) searched for low surface brightness galaxies in the Hubble Ultra Deep Field, with the expectation that, based on Damped Lyman Alpha (DLA) neutral gas column density statistics and the star formation law, a measurable fraction of the sky should be covered by low brightness objects if star formation takes place in DLAs. Their study (as well as the recent extension by Rafelski et al. 2011) shows that star formation activity in DLAs is suppressed by over an order of magnitude (factors of 30-100) with respect to the predictions based on the total gas Schmidt law. Wolfe \& Chen indeed suggest that part of the explanation may be a low molecular fraction in DLAs. More recently, Krumholz et al. (2009a) show that although the observed distribution of column densities in QSO-DLA systems theoretically requires the existence of a significant cold phase, they are inconsistent with the expectation of large molecular fractions. This is simply a reflection of the fact that, given their low metallicities, their densities are not large enough to sustain significant molecular 
fractions. The analysis of the SMC presented here shows that star formation activity is directly proportional to the molecular content.

\section{COMPARISON TO STAR FORMATION MODELS}

How do our results in the SMC compare with predictions from models? The last few years have seen a range of very important theoretical and computational modeling effort concerning the star formation law in galaxies (e.g., Schaye 2004; Blitz \& Rosolowsky 2006; Schaye \& Dalla Vecchia 2008; Robertson \& Kravtsov 2008; Gnedin \& Kravtsov 2010, 2011, to mention just a few). In the following sections we will focus on two recent theoretical models for the dependence of star formation and phase balance on local gas content and other galactic properties, those of Krumholz et al. (2009c; henceforth KMT09) and Ostriker et al. (2010; henceforth OML10). Since the models assume simple geometries, the reader should keep in mind the caveats raised in Section 1.1 about the complex line-of-sight geometry and overall structure of the SMC throughout this section.

The models of KMT09 (summarizing a series of papers) and OML10 adopt different simplifications and focus on different aspects of gas phase balance in the ISM, and are therefore complementary. The KMT09 model adopts the simplification that all neutral gas is gathered into high-density, cold atomicmolecular complexes with surface density $\Sigma_{\text {comp }}$ a factor of $c$ larger than the mean gas surface density $\Sigma_{\text {gas }}$ averaged over large scales (radio observations typically sample scales $\gtrsim 0.3 \mathrm{kpc}$ for galaxies outside the Local Group). The parameter $c$ is not predicted by KMT09, but the comparison with observations suggests $c \sim 5$ on scales $r \sim 750 \mathrm{pc}$, and by definition $c \sim 1$ on scales approaching the size of molecular cloud complexes where $\Sigma_{\text {comp }}$ dominates the surface density of gas (several tens of parsecs to $\sim 100 \mathrm{pc}$ for the Milky Way). Warm, diffuse H I gas is assumed to represent a negligible fraction of the total gas content. This assumption does not in fact appear to be satisfied globally in the SMC, based on our estimated $\mathrm{H}_{2}$ mass of $2.2 \times 10^{7} M_{\odot}$ and an $\mathrm{HI}$ mass of $4.2 \times 10^{8} M_{\odot}$, most of which is believed to be warm (Dickey et al. 2000). The cold $\mathrm{H} \mathrm{I}+\mathrm{H}_{2}$ component, however, probably still dominates over warm $\mathrm{H}_{\mathrm{I}}$ locally. In the KMT09 model, the $\mathrm{H}_{2} / \mathrm{H}_{\mathrm{I}}$ balance within complexes is determined by shielding of dissociating radiation and depends primarily on $Z \Sigma_{\text {comp. Star formation is }}$ assumed to take place exclusively within gas which is $\mathrm{H}_{2}$ rather than $\mathrm{HI}$, at the typical rates and efficiencies observed locally, of a few percent per free-fall timescale. Comparison between our observations and KMT09 are better carried out at the full resolution of the data, at which the clumping factor $c$ should approach unity.

The OML10 model adopts the simplification that all neutral gas is divided between a diffuse component (consisting of both warm and cold atomic gas in mutual pressure equilibrium) and a gravitationally bound component (consisting of cold atomic and molecular gas in unspecified proportion). The amount of diffuse atomic gas is set by the requirement that heating (primarily far-UV (FUV)) balances cooling, with the mean density of the diffuse medium (and hence the cooling rate) set by dynamical equilibrium in the total gravitational potential (provided by stars, gas, and dark matter). Star formation, which produces the FUV, is assumed to occur at a constant rate within the gravitationally bound component. Because only the very densest, highest-column gas within a gravitationally bound cloud actually forms stars and the distribution of densities and columns in highly turbulent clouds depends on temperature but not $\mathrm{H}_{2}$ content, OML10 assumed that the SFR in the gravitationally bound component is independent of the large-scale $\mathrm{H}_{2} / \mathrm{H}$ I proportions within this component, with a depletion time of 2 Gyr. Comparison to observations should be carried out on scales where there is a mix of phases in equilibrium within a resolution element, which we take to be approximately correct at $r \gtrsim 200 \mathrm{pc}$.

\subsection{Comparison to KMT09}

The KMT09 model is very successful at reproducing the composite star-forming properties of local samples of galaxies with only three inputs: surface density of gas, metallicity, and a clumping factor $c \equiv \Sigma_{\text {comp }} / \Sigma_{\text {gas }}$ that approaches unity at high spatial resolution. The first two inputs are directly observable. On the other hand, the clumping factor is poorly constrained in unresolved observations and is unlikely to be constant if star-forming cloud complexes are gravitationally bound entities that are isolated from their environments. The clumping factor is introduced to correct the surface densities observed on large spatial scales to the "true" gas surface density on GMC scales, $\Sigma_{\text {comp }}$. Fortunately for us, on the scale of our SMC observations the clumping factor should be approximately unity. Furthermore, these data make it possible to test the effects of resolution on the measurement of the star formation law in $\mathrm{H}$ I-dominated galaxies.

Figure 3 shows the predictions from KMT09 overlaid on the SMC data. We show the model results for three values of the clumping-factor-metallicity product, $c Z=0.2,1$, and 5 . The former value corresponds to the metallicity of the SMC and a clumping factor of unity, which is to be expected given our spatial resolution of $r \sim 12 \mathrm{pc}$. The latter two values bracket most of the observations originally used to test the model (Krumholz et al. 2009b). Although the model drops off more steeply than the data at low $\Sigma_{\text {gas }}$ and overshoots the observations at high $\Sigma_{\text {gas }}$, the overall agreement between the SMC observations and the $c Z=0.2$ model curve is very reasonable.

KMT09 matches the star formation law observations reasonably well for the most part because of its success at reproducing the observed molecular fraction as a function of column density. Figure 5 shows the distribution of the ratio of molecularto-atomic gas as a function of total gas surface density at scales of $r \sim 200 \mathrm{pc}$, together with the model tracks. Although most lines of sight are atomic-dominated, the model with $c Z=0.2$ does a reasonable job of describing the observations. There is almost no differentiation in the location of different subregions of the SMC in this plot. Most notably, the SW end of the SMC Bar with the largest surface densities (where most star formation takes place) tends to lie preferentially below and to the right of the model, while the NE end of the bar produces a lot of the scatter toward lower column densities. The fact that KMT09 obtains reasonable SFRs as a function of surface density suggests that one of the fundamental assumptions underlying the model, that once gas turns molecular it forms stars with approximately fixed efficiency and rate, is approximately correct.

One important check on KMT09 is whether the clumping factor parameter will explain the SMC data at different resolutions. Most of the molecule-dominated disks used in previous comparisons with the model have metallicities that are approximately solar and are consistent with tracks having $c Z \sim 1-5$, suggesting clumping factor values $c \sim 1-5$ at resolutions of several hundred parsecs. The results of spatially smoothing the SMC data for $\Sigma_{\text {SFR }}$ and $\Sigma_{\text {gas }}$ to resolutions $r \sim 200 \mathrm{pc}$ and 


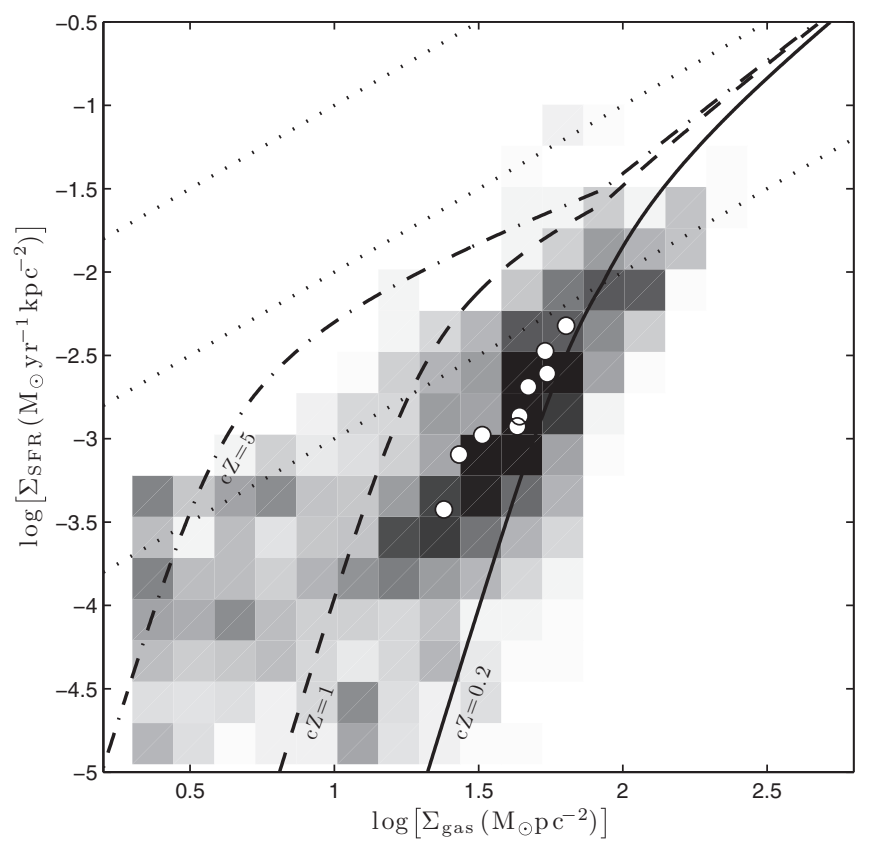

Figure 6. Star formation law in the SMC at different spatial resolutions (compare to the full-resolution results in Figure 3). The gray scale shows the twodimensional distribution of the correlation between $\Sigma_{\text {SFR }}$ and $\Sigma_{\text {gas }}$, as in Figure 3, but now at a resolution $r \sim 200 \mathrm{pc}$. The white circles show the same information at $r \sim 1 \mathrm{kpc}$ resolution. The results of the KMT09 models for different values of the $c Z$ parameter are indicated by the dash-dotted, dashed, and solid lines, as in Figure 3. The dotted lines indicating constant SFE are as in Figures 2 and 3. Degrading the resolution tends to move the star-formation-total-gas relation along a line parallel to the original distribution in Figure 3, rather than shifting to the left following the predictions of KMT09 for different clumping factors.

$r \sim 1 \mathrm{kpc}$ are shown in Figure 6. The gray scale and the white circles represent the results at $200 \mathrm{pc}$ and $1 \mathrm{kpc}$, respectively. We can see that instead of moving from the $c Z=0.2$ track at $r \sim 12 \mathrm{pc}$ to the $c Z \sim 1$ track at $r \sim 1 \mathrm{kpc}$ the points tend to preserve the distribution of the original data, sliding down along a line with slope $1+p \approx 2$ (a fit to the 200 pc resolution points finds $1+p=2.1 \pm 0.2$ ). Thus, even at $r \sim 1 \mathrm{kpc}$ the total gas star formation law points are found more or less along the $c Z=0.2$ track.

Why is the inferred clumping-factor parameter not changing much with resolution? The main reason is that the distribution of $\mathrm{H}$ I surface density is very uniform in the SMC. Thus, going from $r \sim 12$ pc to $r \sim 1 \mathrm{kpc}$ does not dramatically affect the surface density in its central regions. By contrast, the squares and circles in Figure 2 show the effect of going to larger spatial scales on the $\mathrm{H}_{2}$ distribution. The median of the logarithm of $\Sigma_{\mathrm{H} 2}$ on $1 \mathrm{kpc}$ scales decreases by $0.5-0.6$ dex from its value at $r \sim 12 \mathrm{pc}$, about the $x$-axis separation between the $c Z=0.2$ and the $c Z=1$ model tracks in Figure 6. In other words, if the distribution of $\mathrm{H}_{\mathrm{I}}$ were as clumpy as the $\mathrm{H}_{2}$, the change in spatial resolution would shift the points by about the separation between the model tracks along the $x$-axis. Thus, the $\mathrm{H}$ I and $\mathrm{H}_{2}$ gas does not follow a similarly clumped spatial distribution. This calls into question the assumption by KMT09 that the atomic medium is primarily found in shielding envelopes of implicitly cold, dense $\mathrm{H}$ I gas surrounding the $\mathrm{H}_{2}$ gas. Indeed, the observations of Dickey et al. (2000) suggested that the $\mathrm{H}$ I in the SMC is primarily warm, diffuse gas rather than cold, dense clouds.

Note that $c \equiv \Sigma_{\text {comp }} / \Sigma_{\text {gas }}$, so using a constant $c$ over a range of surface densities is strictly incorrect, and this may

\begin{tabular}{ccccccccc}
\multicolumn{8}{c}{$\Sigma_{\text {mol }} \Sigma_{\text {gas }}$} \\
0.1 & 0.2 & 0.3 & 0.4 & 0.5 & 0.6 & 0.7 & 0.8 & 0.9
\end{tabular}
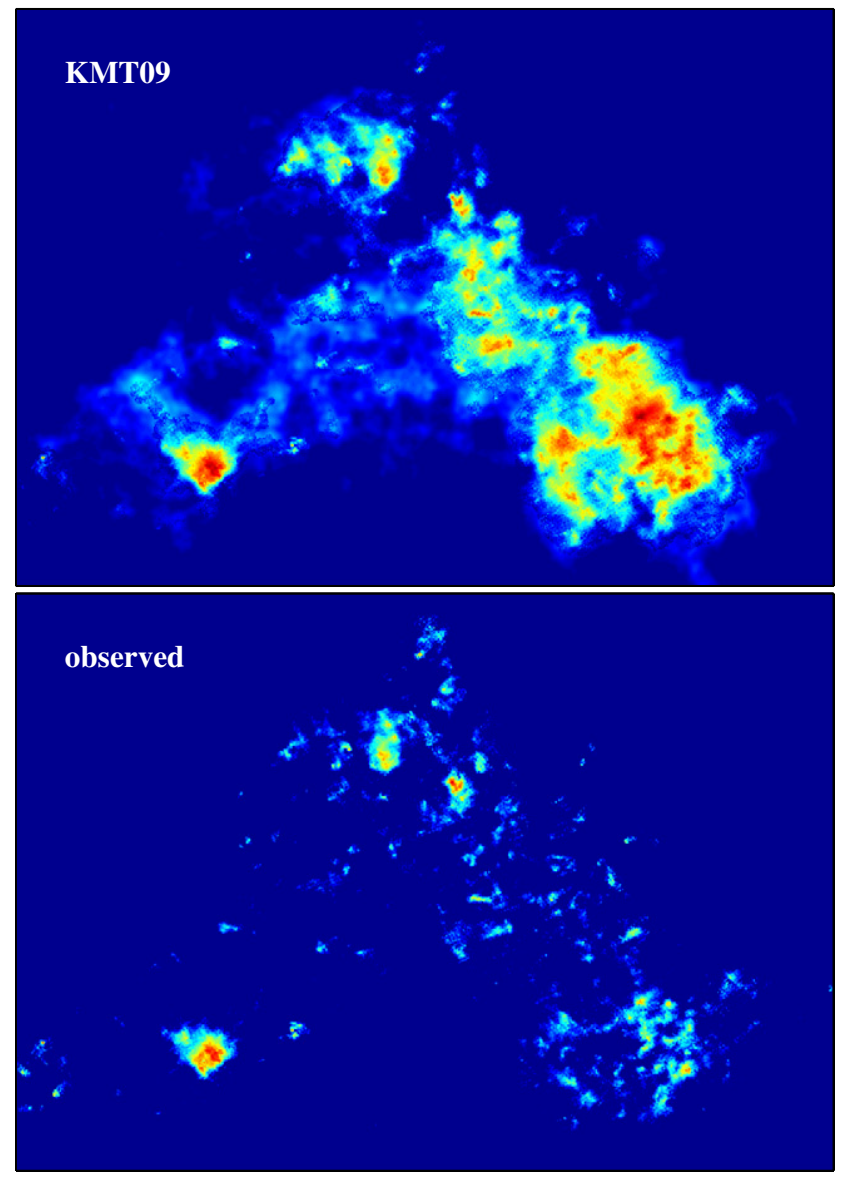

Figure 7. Molecular fraction $\Sigma_{\mathrm{mol}} / \Sigma_{\mathrm{gas}}$ in the SMC, comparison of KMT09 and measurements at $12 \mathrm{pc}$ resolution. The top panel shows the results of KMT09 corresponding to our $\Sigma_{\text {gas }}$ measurements, computed for the metallicity of the SMC and unity clumping factor. The bottom panel shows the measurements obtained from the $\mathrm{H}_{2}$ map presented here (Figure 1) and the $\mathrm{H}$ I observations by Stanimirović et al. (1999). Although the agreement between model and observations is reasonable, KMT09 tends to overpredict (by a factor of $\sim 2$ ) the molecular fraction (and the SFR) at high $\Sigma_{\text {gas }}$. This was already present in Figures 3 and 5 , but it is much more apparent on a linear scale such as that used here. Note that, because of the spatial filtering properties of the algorithm used to produce the $\mathrm{H}_{2}$ map, our measurements are not sensitive to an extended low-level molecular component.

be partially the cause of the apparent slant of the data with respect to the $c Z=0.2$ track, which is present at all resolutions. Imposing $c \sim\left(\Sigma_{\text {gas }}\right)^{-1}$, however, dramatically overcorrects this effect. Fitting the observations requires a softer correction, $c \sim\left(\Sigma_{\text {gas }}\right)^{-0.5}$, suggesting a connection between the surface density of cloud complexes and the density of the surrounding gas on large scales. It is not immediately clear why this should be the case in the context of KMT09.

It is worthwhile to note that although the KMT09 $c Z=0.2$ track is in reasonable overall agreement with the data in Figure 5, there is a noticeable bias toward overpredicting molecular ratios by factors of $\sim 2-3$. Since the discrepancy is of the order of our claimed systematic uncertainty for the $\mathrm{H}_{2}$ map, this is most meaningful in the sense of the relative comparison to OML10. Figure 7 shows the molecular gas fraction $\Sigma_{\text {mol }} / \Sigma_{\text {gas }}$ resulting from applying KMT09 to the observed $\Sigma_{\text {gas }}$ distribution at full resolution (where we know the clumping factor should 
be $c \sim 1$ and $c Z \sim 0.2$ ), compared to our measurements of the same quantity. The discrepancy between the model and observations in the molecular fraction is most apparent for the SW tip of the Bar, which harbors the largest atomic and total gas surface densities. Note that the discrepancy between the data and the $c Z=0.2$ predictions persists although it is somewhat diminished at $r \sim 200 \mathrm{pc}$ (Figure 5), but the model applicable on those scales should have $c>1(c Z>0.2)$ since the molecular complexes are likely unresolved and $\Sigma_{\text {gas }}$ is smaller than $\Sigma_{\text {comp }}$.

\subsection{Comparison to OML10}

The model of OML10 was motivated in part by observations indicating that the SFR and phase balance depend not just on the gas surface density, but also on the density of the stellar component, with the molecular content increasing roughly linearly with the estimated pressure in the ISM (Blitz \& Rosolowsky 2006; Leroy et al. 2008). OML10 explained this empirical result in terms of the simultaneous need to satisfy thermal and dynamical equilibrium in the volume-filling diffuse component. The cooling rate in the diffuse medium is proportional to the gas density, which is controlled by vertical dynamical equilibrium (and hence can be affected by the stellar density if it is large). The heating rate, associated with radiation from high-mass stars in galaxies with active star formation, is proportional to the amount of gas forming stars in gravitationally bound cloud complexes (GBCs). In order to balance cooling and heating, an appropriate partition of gas into diffuse and self-gravitating components is needed. The solutions of the simultaneous thermal/dynamical equilibrium equations were shown by OML10 to agree very well with the radial profiles of star formation in a sample of spiral galaxies. The model of OML10 does not, however, make a prediction for the atomic/molecular balance in the ISM; in Section 4.2.2, we describe an extension of the model that provides this estimate, for comparison to the observations in the SMC.

We use the following implementation of OML10, based on their Equations (10) and (15)-(17),

$$
\begin{gathered}
\Sigma_{\mathrm{diff}}=x \Sigma_{\mathrm{gas}}, \\
\Sigma_{\mathrm{gbc}}=(1-x) \Sigma_{\mathrm{gas}}, \\
\Sigma_{\mathrm{SFR}}=\frac{(1-x) \Sigma_{\mathrm{gas}}}{\tau_{\mathrm{dep}}}, \\
y=\frac{4 \Sigma_{\mathrm{SFR}}}{\Sigma_{\mathrm{SFR}, 0}} \times \frac{Z_{d} / Z_{g}}{1+3.1\left(\Sigma_{\mathrm{gas}} Z_{d} / \Sigma_{0}\right)^{0.365}}, \\
\Sigma_{\mathrm{diff}}=\frac{9.5 M_{\odot} \mathrm{pc}-2 \alpha y}{0.11 \Sigma_{\mathrm{gbc}}+\left[0.011\left(\Sigma_{\mathrm{gbc}}\right)^{2}+\alpha y+100 \alpha f_{\mathrm{w}} \rho_{\mathrm{sd}}\right]^{1 / 2}},
\end{gathered}
$$

where $x$ represents the fraction of gas in the diffuse phase, $\Sigma_{\text {gas }}$, $\Sigma_{\text {diff }}$, and $\Sigma_{\text {gbc }}$ are the large-scale averages of the surface densities of total gas, diffuse phase, and gravitationally bound cold phase, respectively (all in $M_{\odot} \mathrm{pc}^{-2}$ ), and $\rho_{\mathrm{sd}}$ is the midplane volume density of stars plus dark matter (in $M_{\odot} \mathrm{pc}^{-3}$ ). The parameters $\Sigma_{0} \approx 10 M_{\odot} \mathrm{pc}^{-2}, \Sigma_{\mathrm{SFR}, 0} \approx 2.5 \times 10^{-9} M_{\odot} \mathrm{pc}^{-2} \mathrm{yr}^{-1}, \alpha \approx 5$, $f_{\mathrm{w}} \approx 0.5$, and $\tau_{\mathrm{dep}} \approx 2 \times 10^{9} \mathrm{yr}$ are, respectively, the surface density of gas and the SFR at the solar circle, the ratio of totalto-thermal pressure, the fraction of diffuse gas in the warm phase, and the gas depletion time in the gravitationally bound component (as inferred empirically from spiral galaxies). The parameter $y$ is simply the normalized thermal pressure in the model, $y \equiv\left(P_{\mathrm{th}} / k\right) / 3000 \mathrm{~K} \mathrm{~cm}^{-3}$ where $k$ is Boltzmann's constant. For a two-phase ISM, $P_{\text {th }}$ is proportional to the UV heating rate (Wolfire et al. 2003), which is proportional to $\Sigma_{\text {SFR }}$ (as expressed in Equation (8)). Finally, the dust-to-gas ratio and the gas-phase metallicity, both relative to the Milky Way solar circle values, are indicated by $Z_{d}$ and $Z_{g}$. For the SMC we assume $Z_{d} \cong Z_{g}=0.2$ (e.g., Leroy et al. 2007a).

Given a local $\Sigma_{\text {gas }}$ and $\rho_{\text {sd }}$, we substitute Equations (5) to (8) into Equation (9), finding the value of $x$ in the $[0,1]$ interval that satisfies it. The corresponding value of $\Sigma_{\mathrm{SFR}}$ can then be readily found using Equation (7).

As inputs to the model, besides our total surface density gas map, we employ the stellar surface density derived from the SAGE-SMC $3.6 \mu \mathrm{m}$ images (Gordon et al. 2011) using the mass-to-light ratio from Leroy et al. (2008) and the dark matter density profile results from Bekki \& Stanimirović (2009). To deproject the stellar surface density we use a disk scale height of $2 \mathrm{kpc}$. Because the thickness of the SMC is very poorly constrained this is simply a guess. Nonetheless, since the term associated with $\rho_{\text {sd }}$ in Equation (9) is much smaller than that associated with the gas terms, it turns out that the precise value of the scale height has little impact on the final result. Under these assumptions the typical values for the bar and wing regions of the SMC are $\rho_{\mathrm{sd}} \simeq 0.015-0.03 M_{\odot} \mathrm{pc}^{-3}$, with a dark matter contribution $\rho_{\mathrm{dm}} \simeq 0.008 M_{\odot} \mathrm{pc}^{-3}$. As explained in OML10, for near-solar metallicity and GBCs with cloud complexes surface densities $\Sigma_{\text {comp }} \sim 100 M_{\odot} \mathrm{pc}^{-2}$, individual GBCs are well shielded so that $\Sigma_{\mathrm{H} 2} \sim \Sigma_{\mathrm{gbc}}$ and $\Sigma_{\mathrm{HI}} \sim \Sigma_{\mathrm{diff}}$. At low metallicity, however, GBCs may have substantial $\mathrm{H}_{\mathrm{I}}$ envelopes such that $\Sigma_{\mathrm{H} 2}<\Sigma_{\mathrm{gbc}}$ and $\Sigma_{\mathrm{H} \text { I }}>\Sigma_{\text {diff. We will revisit }}$ this issue in Section 4.2.2, obtaining an estimate for the separate $\mathrm{H}$ I and $\mathrm{H}_{2}$ contributions to $\Sigma_{\text {gbc }}$.

We find that following Equations (5)-(9) with the same parameters as adopted by OML10, $\Sigma_{\text {diff }}$ is substantially lower than $\Sigma_{\mathrm{HI}}$ in the SMC, yielding $\Sigma_{\mathrm{gbc}} / \Sigma_{\text {diff }}$ much larger than the observed $\Sigma_{\mathrm{H} 2} / \Sigma_{\mathrm{H} \text { I }}$ ratios. The distribution of the model predictions computed for $\rho_{\text {sd }}$ in the SMC are shown by the gray contour in Figure 5. This result is robust to our choice of $\rho_{\text {sd }}$ and the precise values of $f_{\mathrm{w}}, Z_{d}$, and $\tau_{\mathrm{dep}}$. In essence, total gas pressure (which is $\alpha P_{\text {th }}$ ) is what balances self-gravity, and $P_{\text {th }}$ must also be consistent with the balance between heating and cooling. The equilibrium value of $P_{\text {th }}$ is insensitive to metallicity (Equation (8)) because photoelectric heating is $\propto Z_{d}$ whereas metal cooling is $\propto Z_{g}$. Consequently at the high column densities and pressures present in the SMC, the model requires that most of the gas is driven into the self-gravitating cold phase almost independent of metallicity, in order to generate the radiation field and the corresponding heating needed to attain pressure equilibrium in the diffuse gas. For $\Sigma_{\text {diff }}$ to approach the large values of $\Sigma_{\mathrm{H}_{\mathrm{I}}}$ observed in the SMC would imply large values of the pressure, which necessitates either increases in $P_{\text {th }}$ (and consequently the heating), or a large turbulent factor represented by $\alpha$. Matching the observations with an increase in $\alpha$ requires a very large value, $\alpha \sim 20$, which is likely unrealistic (Burkhart et al. 2010). For the reference value, $\alpha \approx 5$, the predicted surface density of the diffuse phase in the SMC is $\Sigma_{\text {diff }} \lesssim 30 M_{\odot} \mathrm{pc}^{-2}$, in contrast with the higher range of $\Sigma_{\mathrm{H}_{\mathrm{I}}}$ evident in Figure 3.

It is important to realize that this point is pretty much independent of the details of the model. In any scheme where the gas is in pressure equilibrium and the geometry is not 
pathological, sustaining large diffuse-H i surface densities requires a correspondingly large heating term to counterbalance the strong cooling of warm $\mathrm{H}$ I at high pressure and density. The gas heating can be driven by the radiation field, or else dynamical energy input (as in the example of increasing the $\alpha$ parameter) can puff up the disk to reduce its density and cooling rate. In either case, energy and momentum input from star formation needs to be present to provide the support necessary to prevent collapse. To match the typical $\mathrm{H}$ i surface density observed in the SMC at $200 \mathrm{pc}$ resolution, $\Sigma_{\mathrm{H}_{\mathrm{I}}} \sim 60-70 M_{\odot} \mathrm{pc}^{-2}$, would require a thermal pressure $P_{\text {th }} / k \sim 24-32 \times 10^{4} \mathrm{~K} \mathrm{~cm}^{-3}$ in the diffuse phase (assuming $\alpha=5$ ), far in excess of what is observed in the Milky Way plane.

Attaining such pressures requires a large heating term, and equilibrium demands it be balanced by an equally large cooling. Since the cooling in neutral gas is dominated by far-infrared fine-structure lines over a wide range of densities (Wolfire et al. 1995), we expect that the consequence of increased heating in the diffuse medium would be observable. Note that there is evidence that the $\left[\mathrm{C}_{\mathrm{II}}\right] 157.7 \mu \mathrm{m}$ transition is indeed bright in the Magellanic Clouds and other low-metallicity dwarfs (Stacey et al. 1991; Israel et al. 1996; Madden et al. 1997), but those measurements are likely dominated by the dense, selfgravitating phase. Testing this hypothesis will likely require observations of the diffuse phase.

Before proceeding to consider the possibility of enhanced heating in more detail, we note that an increase in the assumed inclination angle $i$ of the SMC's plane would also yield a lower prediction for $\Sigma_{\mathrm{gbc}} / \Sigma_{\mathrm{diff}}$, and lower $P_{\mathrm{th}}$. For the gas-gravity-dominated case, Equation (9) yields $y \sim$ $\left(\Sigma_{\text {gas }} / 9.5 M_{\odot} \mathrm{pc}^{-2}\right)^{2} / \alpha$ for the normalized thermal pressure, while Equations (6)-(8) yield $y \sim \Sigma_{\mathrm{gbc}} /\left(\Sigma_{\mathrm{SFR}, 0} \tau_{\mathrm{dep}}\right)$. If diffuse gas dominates, combining these leads to

$$
\frac{\Sigma_{\mathrm{gbc}}}{\Sigma_{\mathrm{diff}}} \sim \frac{\Sigma_{\mathrm{gbc}}}{\Sigma_{\mathrm{gas}}} \sim \frac{\Sigma_{\mathrm{gas}} \Sigma_{\mathrm{SFR}, 0} \tau_{\mathrm{dep}}}{\left(9.5 M_{\odot} \mathrm{pc}^{-2}\right)^{2} \alpha} .
$$

Taking fiducial parameter values for $\Sigma_{\mathrm{SFR}, 0}, \tau_{\text {dep }}$, and $\alpha$, this yields $\Sigma_{\text {gbc }} / \Sigma_{\text {gas }} \sim \Sigma_{\text {gas }} / 100 \quad M_{\odot} \mathrm{pc}^{-2}$. A reduction of deprojected $\Sigma_{\text {gas }}$ by a factor $\mu$ would lower $\Sigma_{\text {gbc }} / \Sigma_{\text {diff }}$ by the same factor. Thus, if the typical deprojected value of $\Sigma_{\text {gas }}$ were $20 M_{\odot} \mathrm{pc}^{-2}$ rather than $60 M_{\odot} \mathrm{pc}^{-2}$, the predicted $\Sigma_{\mathrm{gbc}} / \Sigma_{\text {diff }}$ would be a factor of $\sim 3$ lower. To reduce $\Sigma_{\mathrm{gbc}} / \Sigma_{\mathrm{diff}}$ to the level $\sim 0.1$ of the molecular-to-atomic ratio would require an inclination correction factor $\mu \sim 0.1$ (corresponding to an almost edge-on inclination $i \sim 85^{\circ}$ ), however, which seems unlikely.

\subsubsection{Enhanced Heating?}

What could be the source of the extra diffuse gas heating in the case of the SMC? In the following we explore the possibility of more effective diffuse gas heating happening at low metallicities. One potential solution is that the local radiation field (relative to the solar neighborhood), $G_{0}^{\prime}$, is enhanced at low metallicities with respect to the corresponding case at $Z=1$. A simplification introduced in the OML10 model, as described in the paper, is to ignore local variations in the propagation of the FUV photons, which determine $G_{0}^{\prime}$ and the heating of the gas. Thus, the radiation field relative to the solar circle in the Milky Way is represented in the heating term (Equation (8)) by the factor $\Sigma_{\mathrm{SFR}} / \Sigma_{\mathrm{SFR}, 0}$. Note that the optical depth to FUV radiation, $\tau_{\mathrm{UV}}$, is determined by dust attenuation. For identical columns of gas, this is lower in a low-metallicity environment because of the lower dust-to-gas ratio $Z_{d}$, such that $\tau_{\mathrm{UV}} \approx 1 / 2 A_{\lambda} / A_{V}\left(N_{\mathrm{H}} / 2 \times 10^{21} \mathrm{~cm}^{-2}\right) Z_{d}$. The escape probability of FUV photons, $\beta_{\mathrm{UV}} \simeq\left(1-e^{-\tau_{\mathrm{UV}}}\right) / \tau_{\mathrm{UV}}$, which in the limit of large $\tau_{\mathrm{UV}}$ is $\beta_{\mathrm{UV}} \sim 1 / \tau_{\mathrm{UV}}$, is consequently higher at lower $Z_{d}$ (for a given $N_{\mathrm{H}}$ ). To account for this effect we can introduce a factor of $\beta_{\mathrm{UV}} \sim 1 / Z_{d}$ in Equation (8), so that the normalized thermal pressure becomes

$$
y=\frac{4 \Sigma_{\mathrm{SFR}}}{\Sigma_{\mathrm{SFR}, 0}} \times \frac{1 / Z_{g}}{1+3.1\left(\Sigma_{\mathrm{gas}} Z_{d} / \Sigma_{0}\right)^{0.365}} .
$$

Interestingly, there is some evidence for an enhanced mean radiation field in the Bar region of the SMC. Modeling of the dust emission suggests that the average $G_{0}^{\prime}$ is $\sim 4-5$ times larger than the local Mathis et al. (1983) radiation field (Sandstrom et al. 2010). An extended warm dust component associated with the Bar is also observed in the recent analysis using Planck data by Planck Collaboration et al. (2011b). The mean radiation field in the Wing by Sandstrom et al. (2010) appears to be approximately Galactic, with the exception of its tip and the star-forming regions located there, despite the large values of $\Sigma_{\mathrm{H}_{\mathrm{I}}}$ present. The modeling of the dust emission, however, is particularly difficult in the Wing, where the signal-to-noise ratio of the observations is lowest.

With Equation (11) replacing Equation (8) in the model the resulting $\Sigma_{\text {diff }}$ reproduces the large observed $\Sigma_{\mathrm{HI}}$ in the SMC using the nominal (Galactic) values for the rest of the parameters, including $\alpha$. A comparison of the predictions of OML10 modified for heating (OML10h henceforth) with our measurements of the molecular fraction is shown in Figures 5 and 8 . OML10h does very well at reproducing the average trends in the data, although local over- or underpredictions persist at the factor-of-two level.

The above approach is perhaps unrealistically simple, and it is likely to overestimate the correction to the local interstellar radiation field due to the diminished FUV extinction at low metallicities. An important fraction of the UV radiation escapes $\mathrm{H}$ II regions through very low extinction lines of sight, and that fact should be taken into account in the FUV propagation. In more general terms, the local interstellar radiation field relative to the solar neighborhood, $G_{0}^{\prime}$, is

$$
G_{0}^{\prime}=\frac{(1-x) \Sigma_{\mathrm{gas}}}{\tau_{\mathrm{dep}} \Sigma_{\mathrm{SFR}, 0}} \frac{f}{f_{0}},
$$

where $f / f_{0}$ is the enhancement factor for the radiation field relative to the solar circle value due to propagation effects, and it includes the escape of radiation from star-forming regions, as well as the propagation through the diffuse gas. This factor can be written as

$$
\begin{aligned}
& \frac{f}{f_{0}}= {\left[\frac{f_{\mathrm{esc}}+\frac{1-e^{-\tau_{\mathrm{gbc}}}}{\tau_{\mathrm{gbc}}}\left(1-f_{\mathrm{esc}}\right)}{f_{\mathrm{esc}, 0}+\frac{1-e^{-\tau_{\mathrm{gbc}}, 0}}{\tau_{\mathrm{gbc}, 0}}\left(1-f_{\mathrm{esc}, 0}\right)}\right] } \\
& \times\left[\frac{1-E_{2}\left(\tau_{\mathrm{diff}} / 2\right)}{1-E_{2}\left(\tau_{\mathrm{diff}, 0} / 2\right)} \frac{\tau_{\mathrm{diff}, 0}}{\tau_{\mathrm{diff}}}\right],
\end{aligned}
$$

where $f_{\text {esc }}$ is the fraction of the UV radiation directly escaping star-forming regions, $E_{2}$ is the second exponential integral function, $\tau_{\mathrm{gbc}}$ and $\tau_{\mathrm{diff}}$ are the optical depth to the UV associated with gravitationally bound clouds and diffuse gas, respectively, and the 0 subscript indicates solar circle Milky Way reference values. The second term in square brackets, as introduced in OML10, assumes that the diffuse gas is a uniform slab with 

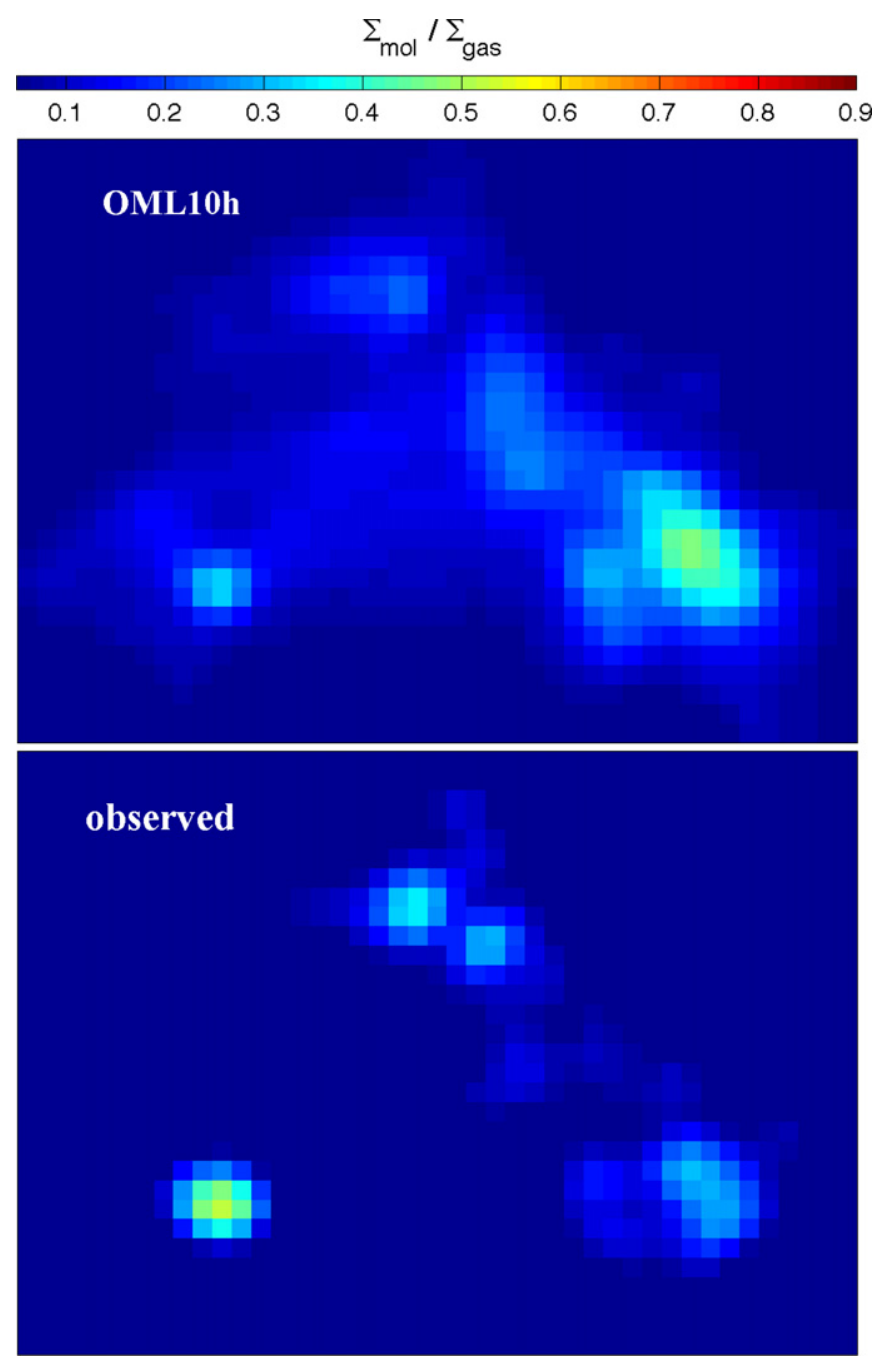

Figure 8. Molecular fraction $\Sigma_{\mathrm{mol}} / \Sigma_{\mathrm{gas}}$ in the SMC, comparison of OML10 and measurements at $200 \mathrm{pc}$ resolution. The top panel shows the results of OML10h, the OML10 model modified to include enhanced heating in the diffuse phase at low metallicities as discussed in Section 4.2.1. The bottom panel shows the measurements obtained from the $\mathrm{H}_{2}$ map presented here (Figure 1) and the H I observations by Stanimirović et al. (1999). Note that, because of the spatial filtering properties of the algorithm used to produce the $\mathrm{H}_{2}$ map, our measurements are not sensitive to an extended low-level molecular component.

total optical depth $\tau_{\text {diff }}=\kappa_{\mathrm{UV}} \Sigma_{\text {diff }}$. Note that the correction factor introduced in Equation (11) is just a simplification of Equation (13) considering only the factor between the first square brackets, due to the gravitationally bound phase, with $f_{\text {esc }}=0$. Although it is possible to reproduce the observations of the SMC using this approach, it requires choosing values for a number of very poorly constrained parameters.

Other poorly constrained parameters in the problem of how the low metallicity affects the diffuse gas heating are the changes in the properties of the dust grains that couple the radiation field to the gas. The smallest carbonaceous dust grains in the ISM, associated with the $2175 \AA$ extinction bump and the mid-infrared aromatic features attributed to PAHs (Draine \& Li 2001; Li \& Draine 2002), are considerably depleted at low metallicities and in particular in the SMC (Madden et al. 2006; Engelbracht et al. 2008; Sandstrom et al. 2010). If these grains dominate the heating, as appears to be the case for the LMC (Rubin et al. 2009), the consequently lower photoelectric heating efficiency could make it more difficult to heat the diffuse gas despite the enhanced radiation field.

We conclude that including systematic heating effects in the diffuse H I gas at low metallicities, because the enhanced radiation escape from the dense star-forming phase offers a viable model, is able to approximately reproduce the data. We will return to whether this is the best solution in Section 4.2.3.

\subsubsection{Is All the H I Diffuse?}

An alternative explanation for the observations of the SMC in the context of the OML10 model is that only a small fraction of the observed $\Sigma_{\mathrm{H}_{\mathrm{I}}}$ is diffuse gas contributing to $\Sigma_{\text {diff }}$, and the remainder of the $\mathrm{HI}_{\mathrm{I}}$ is actually in gravitationally bound structures contributing to $\Sigma_{\text {gbc }}$. Physically, we would expect the H I component of individual GBCs to be the envelopes, and the $\mathrm{H}_{2}$ component the shielded interiors (note that the clumpy structure of clouds means both the "envelope" and "interior" may pervade the whole cloud complex). The relation between $\mathrm{H}_{\mathrm{I}}$ and $\mathrm{H}_{2}$ within GBCs would then be determined by the physics of photodissociation described in the KMT09 model. In principle, it should be possible to test this idea by searching for the kinematical signatures of these gravitationally bound structures in the existing $\mathrm{H}$ I data cubes.

As noted in Section 4.1, we have argued in Section 3.3 that neither Figure 4 nor the results of Dickey et al. (2000) support the scenario that most of the $\mathrm{HI}$ is cold gas in the envelopes of GBCs (which are atomic-molecular cloud complexes in the terminology of KMT09). Nonetheless, it is interesting to further investigate its consequences in the context of the OML10 model, just as we did for the model of KMT09.

To compute the amount of $\mathrm{H}$ I in GBC envelopes we can apply the molecular fraction determination from the KMT09 model to the self-gravitating portion of the gas, and rely on the OML10 model described by Equations (5)-(9) to determine the balance between the diffuse and self-gravitating phases. The equations for the resulting surface densities of $\mathrm{H}_{\mathrm{I}}$ and $\mathrm{H}_{2}$ averaged over large scales are

$$
\begin{gathered}
\Sigma_{\mathrm{H} \mathrm{I}}=\Sigma_{\mathrm{gas}}\left[x+(1-x)\left(1-f_{\mathrm{H} 2}\right)\right], \\
\Sigma_{\mathrm{H} 2}=\Sigma_{\text {gas }}(1-x) f_{\mathrm{H} 2},
\end{gathered}
$$

where $f_{\mathrm{H} 2}=f_{\mathrm{H} 2}\left(\Sigma_{\text {comp }}, Z\right)$ is determined through Equation (2) in KMT09. It is a function of the surface density of the cloud, $\Sigma_{\text {comp }}$, and the metallicity $Z$ relative to the Milky Way, which we take to be $Z=Z_{d}=Z_{g}$. We take $\Sigma_{\text {comp }}$ to be the larger of $\Sigma_{\mathrm{gbc}}=(1-x) \Sigma_{\mathrm{gas}}$ and $100 M_{\odot} \mathrm{pc}^{-2}$. The latter is the approximate value for the surface density of resolved molecular clouds in nearby galaxies (Bolatto et al. 2008). Thus, this model assumes that GBCs have at least this surface density, and lower surface densities are the result of beam dilution in the observations. The fraction of all $\mathrm{H}$ I found in the gravitationally bound phase, $f_{\mathrm{HI}}^{\mathrm{gbc}}$, will then be

$$
f_{\mathrm{HI}}^{\mathrm{gbc}}=\frac{(1-x)\left(1-f_{\mathrm{H} 2}\right)}{\left[x+(1-x)\left(1-f_{\mathrm{H} 2}\right)\right]} .
$$

Because this model extends OML10 by incorporating the photodissociation model of KMT09 for comparison to observations of the $\mathrm{H}$ I abundance, we henceforth refer to it as OML10p. The behavior of the Hi component in this model is shown in Figure 9. After accounting for the $\mathrm{H}_{\mathrm{I}}$ in the gravitationally bound gas through the inclusion of the photodissociation term 

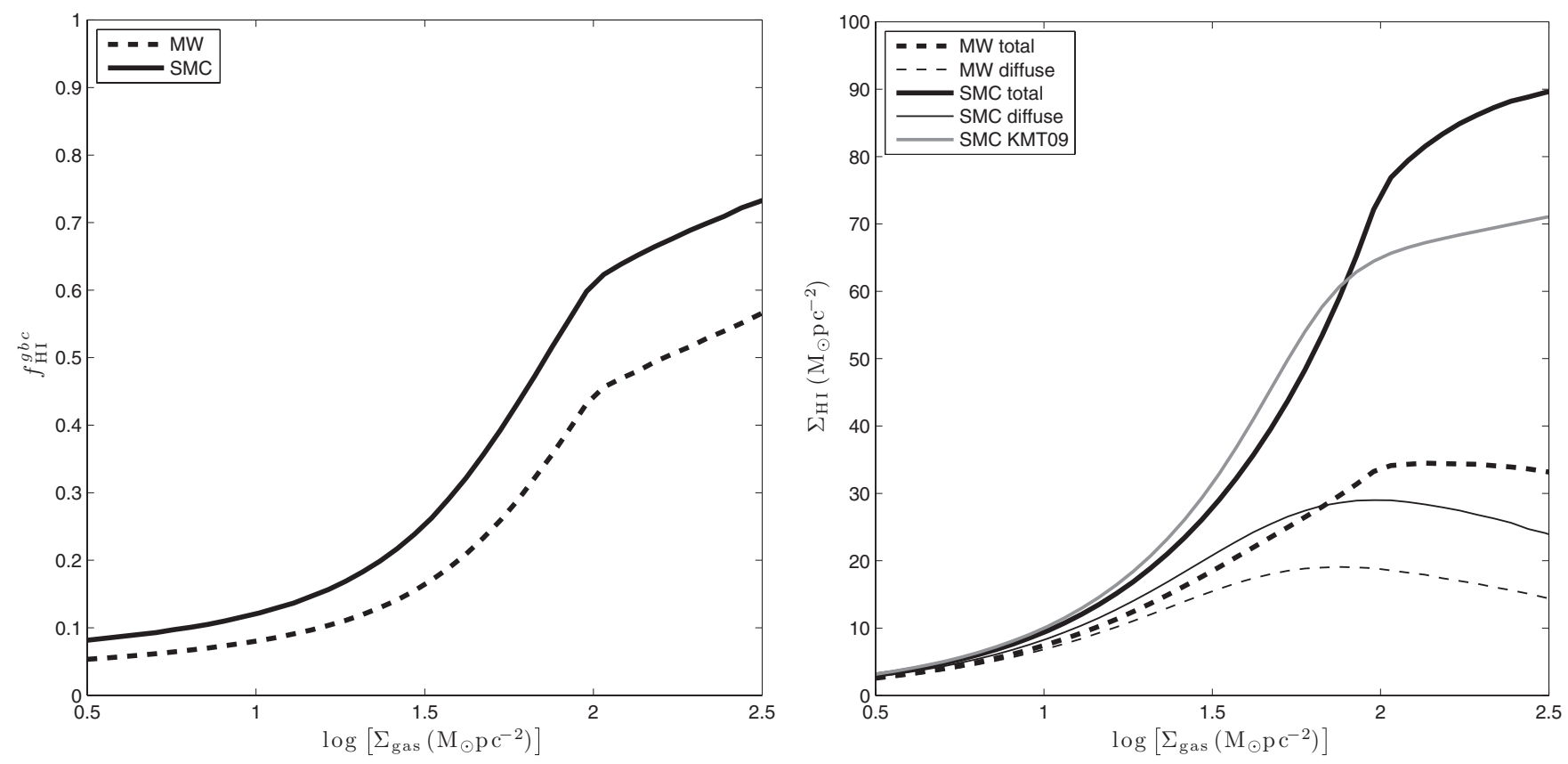

Figure 9. Behavior of the atomic component in the OML10p model described in Section 4.2.2 and comparison to the diffuse component in OML10, under typical conditions for the solar circle in the Milky Way $\left(\rho_{\mathrm{sd}}=0.05 M_{\odot} \mathrm{pc}^{-3}\right.$ and $\left.Z=1\right)$ and the SMC $\left(\rho_{\mathrm{sd}}=0.02 M_{\odot} \mathrm{pc}^{-3}\right.$ and $\left.Z=0.2\right)$. Left: fraction of the total $\mathrm{HI}$ surface density arising from the gravitationally bound phase according to Equation (16). The diffuse and the gravitationally bound phases make approximately equal contributions to $\Sigma_{\mathrm{H}_{\mathrm{I}}}$ at surface densities of approximately $160(70) M_{\odot} \mathrm{pc}^{-2}$ for typical conditions in the local Milky Way (SMC). Right: surface density of $\mathrm{HI}$ as a function of total gas surface density in OML10p, with the prediction of KMT09 for comparison. With the contribution of H I from the gravitationally bound phase, it is possible to match the typical $\Sigma_{\mathrm{H}_{\mathrm{I}}} \approx 70 M_{\odot} \mathrm{pc}^{-2}$ observed in the SMC without an increase in the heating term.

from KMT09, the surface density of atomic gas saturates at $\Sigma_{\mathrm{HI}} \sim 90 M_{\odot} \mathrm{pc}^{-2}$ under the typical conditions found in the SMC instead of the $\Sigma_{\mathrm{HI}_{I}} \lesssim 30 M_{\odot} \mathrm{pc}^{-2}$ stemming from the diffuse component alone. In fact, the H I associated with the gravitationally bound gas becomes the dominant contributor to $\Sigma_{\mathrm{HI}}$ for surface densities $\Sigma_{\text {gas }} \gtrsim 70 M_{\odot} \mathrm{pc}^{-2}$ under SMC conditions. Inclusion of the modifications to the heating term discussed in Section 4.2.1 mostly affect this threshold surface density, and increase the saturation value of $\Sigma_{\mathrm{H}_{\mathrm{I}}}$ only moderately $\left(\Sigma_{\mathrm{HI}} \lesssim 150 M_{\odot} \mathrm{pc}^{-2}\right.$ using Equation (11), for example).

Figure 10 shows the distribution of the molecular-to-atomic ratio $\Sigma_{\mathrm{H} 2} / \Sigma_{\mathrm{HI}}$ in the SMC according to this model, compared with KMT09 and the distribution of the measurements at $200 \mathrm{pc}$ resolution as in Figure 5. The similarity at $\Sigma_{\text {gas }} \gtrsim 100 M_{\odot} \mathrm{pc}^{-2}$ between OML10p and the KMT09 track corresponding to $c Z=0.2$ is simply due to the fact that the $\mathrm{H}$ a arising from the diffuse component is not dominant at these surface densities. The inclusion of the diffuse $\mathrm{H}_{\mathrm{I}}$ in the $\Sigma_{\mathrm{HI}}$ budget is responsible for the displacement down from the KMT09 track by a factor of $\sim 0.6$.

We conclude that including the effects of photodissociation into the calculations to compare the predictions of OML10 to the $\mathrm{HI}$ and $\mathrm{H}_{2}$ data is physically motivated and potentially offers an alternative explanation for the observed low values of the $\Sigma_{\mathrm{H} 2} / \Sigma_{\mathrm{H} \text { I }}$ ratio. As we discuss in the next section, however, we think this is not the dominant consideration in explaining the data.

\subsubsection{Consequences for Star Formation}

We have mentioned possible tests of these ideas looking for evidence for enhanced heating or $\mathrm{H} \mathrm{I}$ in the GBC phase using (1) further modeling of dust observations to determine the radiation field, (2) observations of diffuse ISM coolants such as [C II], and (3) searching for kinematic signatures of gravitationally

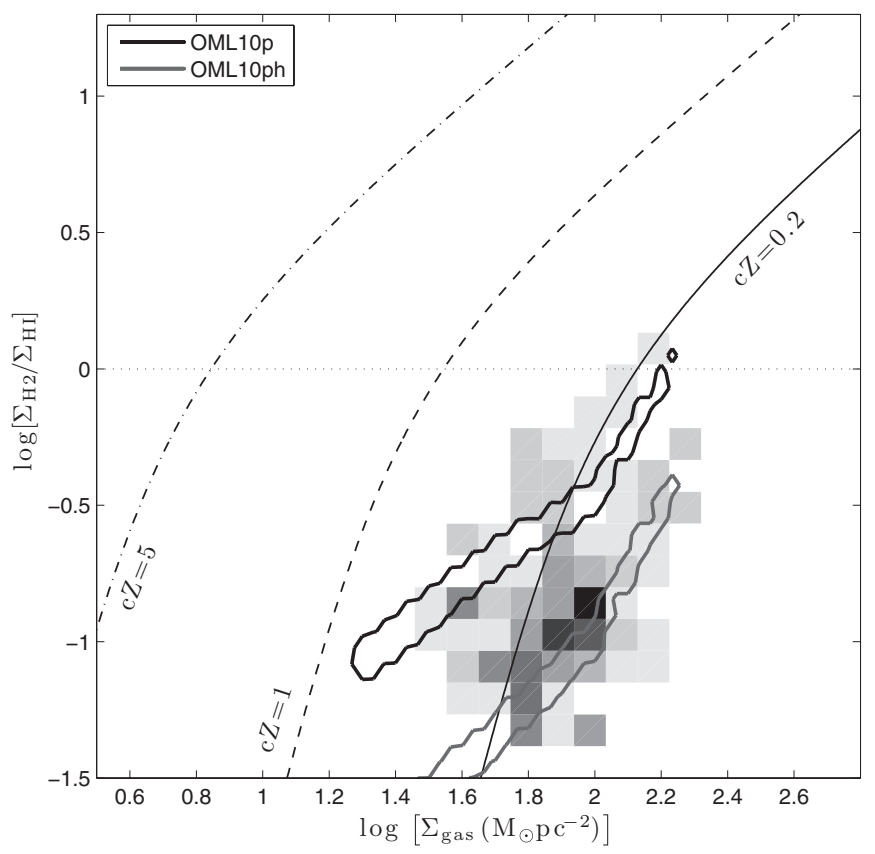

Figure 10. Ratio of molecular to atomic surface densities in the SMC compared to model calculations. The thick black contours show the predictions of the OML10p model discussed in Section 4.2.2, where we include the photodissociation of $\mathrm{H}_{2}$. In this model, $\Sigma_{\mathrm{H}_{\mathrm{I}}}$ in the SMC has a dominant contribution from $\mathrm{H}$ in the gravitationally bound phase. The prediction of the OML10ph model, which includes both photodissociation and enhanced heating in the diffuse phase (see Section 4.2.4), is also shown. Lines from KMT09 are the same as in Figure 5.

bound HI. It should be possible, however, to make an effort to distinguish between these enhanced-heating and standard heating variations of the OML10 model by their impact on the star formation law. 


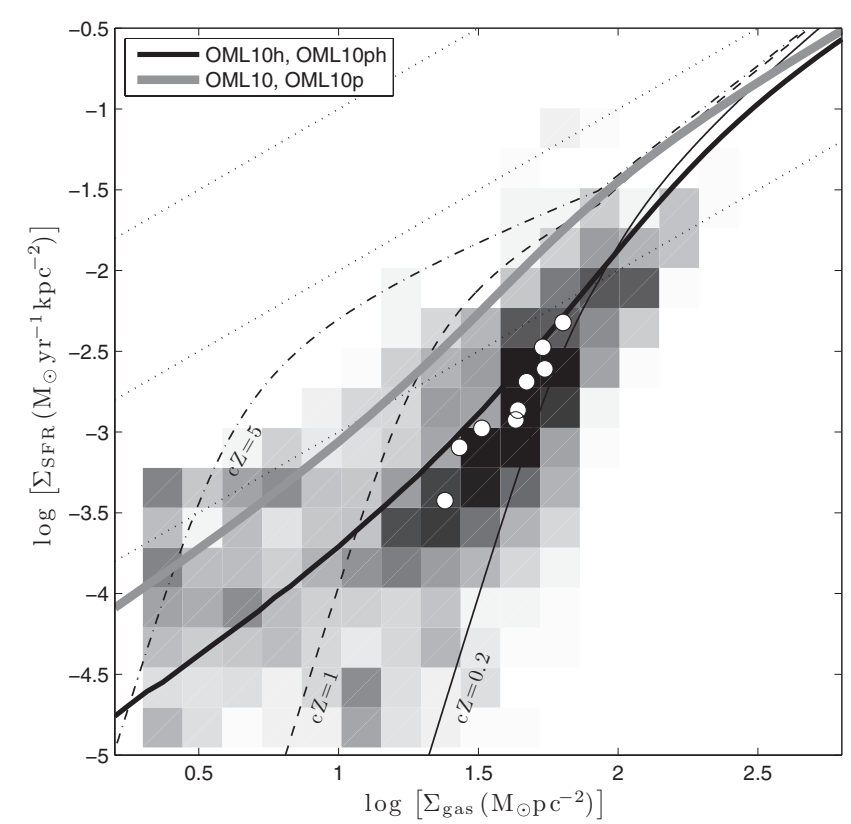

Figure 11. Comparison of OML10 predictions and total gas star formation law observations in the SMC. The thick solid black and gray lines show the results of the enhanced-heating (OML10h, OML10ph) and original-heating (OML10, OML10p) versions of the model. As in Figure 6 the gray scale and white circles represent the SMC observations smoothed to a resolution of $200 \mathrm{pc}$ and $1 \mathrm{kpc}$, respectively. The KMT09 tracks and the constant gas depletion time lines are also the same as in Figure 6.

In the context of OML10, all the gas in the gravitationally bound component could potentially contribute to star formation, although due to the turbulent dynamics within GBCs, only a tiny fraction ( $\sim 1 \%$ per free-fall time) is dense (and cold) enough that it actually collapses and forms stars. In the variant of the model with enhanced heating, OML10h, the gravitationally bound phase is depressed to allow more diffuse $\Sigma_{\mathrm{HI}}$. In the extension of the model that includes an estimate for the contribution to $\Sigma_{\mathrm{HI}}$ from photodissociated envelopes of GBCs, OML10p, the gravitationally bound component is about as abundant as at higher metallicities, and the low observed $\Sigma_{\mathrm{H} 2} / \Sigma_{\mathrm{H} \text { I }}$ ratio is explained by limited shielding of the gravitationally bound gas at low metallicity (as for the dense atomic-molecular complexes in KMT09). As a consequence of the higher abundance of gravitationally bound gas, the SFR expected in OML10p at a fixed gas surface density is significantly higher than in OML10h.

Figure 11 shows the SFR predicted by OML10 with and without additional heating (taking $\rho_{\mathrm{sd}}=0.02 M_{\odot} \mathrm{pc}^{-3}$ and $Z=0.2$ for the SMC), compared to the observations smoothed to $200 \mathrm{pc}$ and $1 \mathrm{kpc}$ resolution and to the KMT09 model, as in Figure 6. Because the specific SFR in GBCs is assumed to be insensitive to their exact atomic/molecular balance, there is no difference in the predicted SFR between OML10 and OML10p. Similarly, OML10h and OML10ph (see below) have the same $\Sigma_{\text {SFR }}$ because they have the same $\Sigma_{\text {gbc }}$ for a given $\Sigma_{\text {gas }}$. The predictions for $\Sigma_{\text {SFR }}$ from OML10, OML10p, and KMT09 with $c=5$ are quite similar for $\Sigma_{\text {gas }} \gtrsim 20 M_{\odot} \mathrm{pc}^{-2}$ because for all of these models in this range of $\Sigma_{\mathrm{gas}}$, the majority of gas is concentrated in cold, dense, star-forming atomicmolecular complexes with $\Sigma_{\text {comp }} \gtrsim 100 M_{\odot} \mathrm{pc}^{-2}$. These models overpredict the $\Sigma_{\mathrm{SFR}}$ observations by about a factor of six. On the other hand, OML10h (and OML10ph) do a very good job of matching not only the average level of SFR activity but also the slope of the $\Sigma_{\mathrm{SFR}}-\Sigma_{\text {gas }}$ relation, despite the simplicity of the heating correction.

\subsubsection{A Unified Model}

It is possible to think of further modifications to OML10. For example, we can extend the enhanced-heating model to include an estimate of the atomic/molecular balance within GBCs; we refer to this as OML10ph. As noted above, this will produce the same $\Sigma_{\mathrm{SFR}}-\Sigma_{\text {gas }}$ results as the OML10h model as it has the same fraction of gravitationally bound gas, but it will yield a molecular-to-atomic ratio $\Sigma_{\mathrm{H} 2} / \Sigma_{\mathrm{H}}$ a factor of $\sim 2.8$ times lower than OML10p (equivalent to a displacement of $\sim 0.45$ in the logarithm, as shown in Figure 10). Such a model is not forbidden by the observations, and in fact it arguably reproduces better the lower values in the distribution of molecular-to-atomic ratios than the model without enhanced heating. In model OML10ph, it is also possible to compute the fraction of $\mathrm{HI}$ gas associated with the gravitationally bound component, $f_{\mathrm{HI}}^{\text {gbc }}$, which has a value $f_{\mathrm{HI}}^{\mathrm{gbc}} \sim 19 \%$ at a surface density $\Sigma_{\mathrm{gas}}=100 M_{\odot} \mathrm{pc}^{-2}$, very similar to the $15 \%$ cold $\mathrm{H}$ I fraction measured by Dickey et al. (2000).

\section{SUMMARY AND CONCLUSIONS}

We present a detailed analysis of the correlation between the atomic and molecular gas phases in the SMC, and the star formation activity. This is the first time such analysis has been done at high spatial resolution in a galaxy of such a low metallicity. We use modeling of the Spitzer dust continuum observations (Bolatto et al. 2007; Gordon et al. 2011) to calculate the $\mathrm{H}_{2}$ surface densities on scales of $\theta \sim 40^{\prime \prime}$ or $r \sim 12 \mathrm{pc}$, together with observations of the H I (Stanimirović et al. 1999) and $\mathrm{H} \alpha$ (Smith \& The MCELS Team 1999) distributions (Figure 1). An important caveat to keep in mind when considering the interpretations of the results is the complexity of the $\mathrm{H}$ I distribution in the SMC, referred to in Section 1.1. Another important caveat are the uncertainties involved in the production of the $\mathrm{H}_{2}$ map, which we discuss in Section 2.1.

We find that, in the regions where we detect $\Sigma_{\mathrm{H} 2}$, we measure a typical molecular gas depletion time $\tau_{\text {dep }}^{\mathrm{mol}} \sim 7.5 \mathrm{Gyr}$ on scales $r \sim 12 \mathrm{pc}$ with a factor of 3.5 uncertainty accounting for the scatter and the systematics associated with our $\mathrm{H}_{2}$ map as well as the geometry of the source (Figure 2). The depletion time shortens when measured on larger spatial scales. On scales $r \sim 200 \mathrm{pc}$ (a typical scale over which molecular gas and $\mathrm{H} \alpha$ emission are well correlated in galaxies) and $r \sim 1 \mathrm{kpc}$ (a typical scale for extragalactic studies) we measure $\tau_{\mathrm{dep}}^{\mathrm{mol}} \approx 1.6 \mathrm{Gyr}$. The molecular depletion time for the SMC as a whole is shorter, $\tau_{\text {dep }}^{\text {mol }} \sim 0.6 \mathrm{Gyr}$, due to a large component of extended low level $\mathrm{H} \alpha$ emission. These results are consistent with the typical depletion time of $\tau_{\text {dep }}^{\mathrm{mol}} \sim 2$ Gyr observed in normal disks on kpc scales (Bigiel et al. 2008, 2011). Consequently, the relation between molecular gas and star formation activity appears to be at most only weakly dependent on metallicity. This finding suggests that the molecular content can be used to infer the star formation activity (and vice versa) even in galaxies that are chemically primitive and deficient in heavy elements.

We also measure the relation between star formation and total gas surface density, which is dominated by H i over most of the SMC (Figure 3). We find that the relation is similarly steep in the SMC $(1+p=2.2 \pm 0.1)$ and in the outer disks of normal galaxies $(1+p \approx 2$; Bigiel et al. 2010b), but it is displaced toward much larger surface densities. In the SMC the $\mathrm{HI}_{\mathrm{I}}$ surface density reaches values as high as $\Sigma_{\mathrm{H}_{\mathrm{I}}} \sim 100 M_{\odot} \mathrm{pc}^{-2}$, while in most galaxies $\Sigma_{\mathrm{H}} \lesssim 10 M_{\odot} \mathrm{pc}^{-2}$. As a consequence, 
the use of the standard total gas to SFR relation dramatically overpredicts the star formation activity over much of this source. This finding supports the explanation that DLAs are deficient in star formation because of very low molecular fractions (Wolfe \& Chen 2006). The gas (or HI) depletion time for the SMC is approximately a Hubble time, $\tau_{\text {dep }}^{\text {gas }} \approx 11.8 \mathrm{Gyr}$.

We compare our results with the recent analytical models by Krumholz et al. (2009c, KMT09) and Ostriker et al. (2010, OML10). For high-resolution observations, we find that KMT09 are very successful at reproducing the displacement in the relation between $\Sigma_{\text {gas }}$ and $\Sigma_{\mathrm{SFR}}$ toward higher surface densities (Figure 3 ). We also find that with a clumping factor $c$ of unity it approximately predicts the observed relation between the molecular-to-atomic ratio $\Sigma_{\mathrm{H} 2} / \Sigma_{\mathrm{H}_{\mathrm{I}}}$ and $\Sigma_{\text {gas }}$ (Figure 5), although on a linear scale it becomes apparent that there is a systematic overprediction at high $\Sigma_{\text {gas }}$ by $0.3-0.5$ dex (Figure 7). The slope of $\Sigma_{\text {SFR }}$ versus $\Sigma_{\text {gas }}$ predicted by KMT09 for fixed $c Z$ is steeper than the relation observed in the SMC, both at the observed 12 $\mathrm{pc}$ resolution and when the data are averaged over $200 \mathrm{pc}$ and $1 \mathrm{kpc}$ scales (Figures 3 and 6). Given that KMT09 co-locate $\mathrm{H} \mathrm{I}$ and $\mathrm{H}_{2}$ within the same dense cloud complexes, a strong relation between $\Sigma_{\mathrm{H}_{\mathrm{I}}}$ and $\Sigma_{\mathrm{SFR}}$ would be expected at $200 \mathrm{pc}$ scales, but this is not seen in the observations (Figure 4). We test the effect of smoothing the observations to larger spatial scales and find that changing the clumping parameter in the KMT09 model does not match the effect of smoothing the observations (Figure 6). The bulk of the total gas surface densities in the SMC stay approximately constant for spatial scales in the $r \sim 12 \mathrm{pc}-1 \mathrm{kpc}$ range. This suggests that much of the $\mathrm{HI}$ is in a warm, diffuse component, which stands in contrast to the starting assumption of KMT09 that the H I consists primarily of cold gas concentrated in the envelopes of dense atomicmolecular complexes, with the warm $\mathrm{H}$ I a small fraction of the total ISM mass.

In comparing the observations to the predictions of OML10, we find that the expected $\Sigma_{\text {gbc }} / \Sigma_{\text {diff }}$ is very large in comparison to the observed $\Sigma_{\mathrm{H} 2} / \Sigma_{\mathrm{H} \text { I }}$ in the SMC (Figure 5) if we adopt the same heating efficiency and turbulence parameters for thermal/dynamical equilibrium as in typical large spirals with $Z \sim 1$. In other words, the model conspicuously overpredicts the molecular fraction in the SMC for the default parameters. This motivates us to investigate variations and extensions of the OML10 model. In the first variation, which we call OML10h, we introduce a metallicity dependence in the ratio of gas heating to star formation that produces enhanced heating at low metallicities. This modification is based on the idea that a lower dust-to-gas ratio allows UV to escape and travel farther from star-forming regions, potentially raising $G_{0}^{\prime}$ significantly in the diffuse phase. An enhanced $G_{0}^{\prime}$ leads to increased heating in the diffuse medium, permitting thermal equilibrium of warm $\mathrm{H}$ I at high pressure. Without enhanced heating, warm gas at the typical thermal pressure in the SMC $\left(P_{\text {th }} / k \sim 3 \times 10^{4} \mathrm{~K} \mathrm{~cm}^{-3}\right.$ for $\Sigma_{\mathrm{H}_{\mathrm{I}}} \sim 65 M_{\odot} \mathrm{pc}^{-2}$ ) would cool very quickly. Enhanced heating changes the balance between the diffuse and self-gravitating phases of the gas. As a consequence the self-gravitating phase is less abundant than at normal metallicities under similar conditions for $\Sigma_{\text {gas }}$. This model does a very reasonable job of matching the $\Sigma_{\mathrm{H} 2} / \Sigma_{\mathrm{H}_{\mathrm{I}}}$ derived for the SMC (Figures 5), although there are still local discrepancies at the level of $\sim \pm 0.3$ dex.

As an extension of OML10, which we term OML10p, we do not change the balance between the diffuse and self-gravitating components, but add an accounting for the abundance of $\mathrm{H}_{\mathrm{I}}$ within gravitationally bound clouds, based on the photodisso- ciation formalism of KMT09. The $\mathrm{H}$ i is split between the diffuse and the self-gravitating phases, and under the typical SMC conditions, half of the H I would be self-gravitating. The photodissociation calculation for the GBC component can also be applied to the enhanced-heating model; we denote this as model OML10ph. Model OML10ph shares the reduced abundance of the self-gravitating phase with model OML10h and includes a contribution to $\mathrm{H}$ I that originates in cold self-gravitating clouds. Note that in adding the photodissociation estimate for GBCs, we do not change the predicted SFR. Thus, models OML10 and OML10p have the same $\Sigma_{\text {SFR }}$ versus $\Sigma_{\text {gas }}$ as each other, as do models OML10h and OML10ph (Figure 11).

When compared to the observed $\Sigma_{\mathrm{H} 2} / \Sigma_{\mathrm{H}_{\mathrm{I}}}$ in the SMC, model OML10p slightly overpredicts the molecular ratio $(\sim 10 \%-30 \%$ for the model versus $\sim 5 \%-15 \%$ for the data; see Figure 10 ). For the typical parameters of the SMC, $\sim 30 \%-60 \%$ of the total H I would be in GBCs for model OML10p (Figure 9). Although this is less than in KMT09 (which puts all of the $\mathrm{H}_{\mathrm{I}}$ in the envelopes of cold, dense cloud complexes), to some extent it shares the difficulty that in observations $\mathrm{HI}_{\mathrm{I}}$ is not correlated with star formation activity (Figure 4). Model OML10ph, with a lower abundance of self-gravitating gas and hence $\Sigma_{\mathrm{H} 2}$ due to enhanced heating, follows the observed $\Sigma_{\mathrm{H} 2} / \Sigma_{\mathrm{H}_{\mathrm{I}}}$ magnitude and slope better than model OML10p (Figure 10). OML10ph also has a lower ratio of cold to warm H i than OML10p or KMT09, consistent with the observations of Dickey et al. (2000). When compared to star formation in the SMC, the enhanced-heating models (OML10h, OML10ph) fit the relation between $\Sigma_{\text {SFR }}$ and $\Sigma_{\text {gas }}$ much better than the models that adopt the same heating efficiency as higher-metallicity spirals (OML10, OML10p), as shown in Figure 11.

In essence, the observed SFR requires that the eligible cold, self-gravitating gas (whether $\mathrm{H}_{\mathrm{I}}$ or $\mathrm{H}_{2}$ ) is less abundant at lower metallicities, otherwise we would expect larger SFRs for the observed $\Sigma_{\text {gas }}$ surface densities. The diminished abundance of the cold self-gravitating phase is supported by observations of the distribution of $\mathrm{H}_{\mathrm{I}}$ temperatures (Dickey et al. 2000) and by the relative uniformity of $\Sigma_{\mathrm{H}_{\mathrm{I}}}$ when averaged at different scales. The possibility of an enhanced radiation field that could maintain warm, diffuse $\mathrm{H}$ I at high surface density and pressure is suggested by recent dust emission modeling (Sandstrom et al. 2010). Further observational confirmation of enhanced heating could come from observations of the cooling in the diffuse atomic gas. Further characterization of the fraction of cold atomic gas and its spatial distribution in both Magellanic Clouds using $\mathrm{H}$ i absorption/emission techniques would also be extremely valuable in constraining the models.

We thank Chris McKee, Mark Krumholz, Andrey Kravtsov, and Nick Gnedin for enlightening and stimulating discussions at several stages of this research, and Jean-Philippe Bernard as well as the anonymous referee for their comments on the manuscript. A.B. and K.J. acknowledge partial support from grants NASA JPL-1314022, NSF AST-0838178, NSF AST-0955836, as well as a Cottrell Scholar award from the Research Corporation for Science Advancement. E.C.O. acknowledges support from grant NSF AST-0908185. M.R. acknowledges support from FONDECYT(CHILE) grant 1080335 and is supported by the Chilean Center for Astrophysics FONDAP 15010003. A.B. also acknowledges travel support from FONDECYT(CHILE) grant 1080335. This research has made use of NASA's Astrophysics Data System. P.F.W. acknowledges support from grant NSF AST-098566. The National Radio Astronomy Observatory is 
a facility of the National Science Foundation operated under cooperative aggreement by Associated Universities, Inc.

\section{REFERENCES}

Bekki, K., \& Stanimirović, S. 2009, MNRAS, 395, 342

Bernard, J. P., Abergel, A., Ristorcelli, I., et al. 1999, A\&A, 347, 640

Bigiel, F., Bolatto, A. D., Leroy, A. K., et al. 2010a, ApJ, 725, 1159

Bigiel, F., Leroy, A., Walter, F., et al. 2008, AJ, 136, 2846

Bigiel, F., Leroy, A., Walter, F., et al. 2010b, AJ, 140, 1194

Bigiel, F., Leroy, A. K., Walter, F., et al. 2011, ApJ, 730, L13

Blanc, G. A., Heiderman, A., Gebhardt, K., Evans, N. J., \& Adams, J. 2009, ApJ, 704,842

Blitz, L., Fukui, Y., Kawamura, A., et al. 2007, in Protostars and Planets V, ed. B. Reipurth, D. Jewitt, \& K. Keil (Tucson, AZ: Univ. Arizona Press), 81 Blitz, L., \& Rosolowsky, E. 2006, ApJ, 650, 933

Bloemen, J. B. G. M., Deul, E. R., \& Thaddeus, P. 1990, A\&A, 233, 437

Boissier, S., Prantzos, N., Boselli, A., \& Gavazzi, G. 2003, MNRAS, 346, 1215

Bolatto, A. D., Jackson, J. M., \& Ingalls, J. G. 1999, ApJ, 513, 275

Bolatto, A. D., Leroy, A. K., Rosolowsky, E., Walter, F., \& Blitz, L. 2008, ApJ, 686, 948

Bolatto, A. D., Simon, J. D., Stanimirović, S., et al. 2007, ApJ, 655, 212

Bot, C., Boulanger, F., Lagache, G., Cambrésy, L., \& Egret, D. 2004, A\&A, 423, 567

Boulanger, F., Abergel, A., Bernard, J., et al. 1996, A\&A, 312, 256

Burkhart, B., Stanimirović, S., Lazarian, A., \& Kowal, G. 2010, ApJ, 708, 1204

Calzetti, D., Kennicutt, R. C., Engelbracht, C. W., et al. 2007, ApJ, 666, 870

Caplan, J., Ye, T., Deharveng, L., Turtle, A. J., \& Kennicutt, R. C. 1996, A\&A, 307,403

Dame, T. M., Hartmann, D., \& Thaddeus, P. 2001, ApJ, 547, 792

Dickey, J. M., Mebold, U., Stanimirović, S., \& Staveley-Smith, L. 2000, ApJ, 536,756

Dobashi, K., Bernard, J., Kawamura, A., et al. 2009, AJ, 137, 5099

Draine, B. T., Dale, D. A., Bendo, G., et al. 2007, ApJ, 663, 866

Draine, B. T., \& Li, A. 2001, ApJ, 551, 807

Dufour, R. J. 1984, in IAU Symp. 108, Structure and Evolution of the Magellanic Clouds, ed. S. van den Bergh \& K. S. D. de Boer (Dordrecht: Reidel), 353

Engelbracht, C. W., Rieke, G. H., Gordon, K. D., et al. 2008, ApJ, 678, 804

Flagey, N., Noriega-Crespo, A., Boulanger, F., et al. 2009, ApJ, 701, 1450

Fukui, Y., Mizuno, N., Yamaguchi, R., et al. 1999, PASJ, 51, 745

Gardan, E., Braine, J., Schuster, K. F., Brouillet, N., \& Sievers, A. 2007, A\&A, 473,91

Glover, S. C. O., \& Mac Low, M. 2011, MNRAS, 412, 337

Gnedin, N. Y., \& Kravtsov, A. V. 2011, ApJ, 728, 88

Gnedin, N. Y., \& Kravtsov, A. V. 2010, ApJ, 714, 287

Gordon, K. D., Meixner, M., Meade, M., et al. 2011, AJ, in press (arXiv:1107.4312)

Harris, J., \& Zaritsky, D. 2004, AJ, 127, 1531

Haschke, R., Grebel, E. K., \& Duffau, S. 2011, AJ, 141, 158

Heiles, C., \& Troland, T. H. 2003, ApJ, 586, 1067

Heyer, M., Krawczyk, C., Duval, J., \& Jackson, J. M. 2009, ApJ, 699, 1092

Hilditch, R. W., Howarth, I. D., \& Harries, T. J. 2005, MNRAS, 357, 304

Hoopes, C. G., Walterbos, R. A. M., \& Rand, R. J. 1999, ApJ, 522, 669

Hughes, A., Wong, T., Ott, J., et al. 2010, MNRAS, 406, 2065

Israel, F. P. 1997, A\&A, 328, 471

Israel, F. P., de Graauw, T., van de Stadt, H., \& de Vries, C. P. 1986, ApJ, 303, 186

Israel, F. P., Johansson, L. E. B., Lequeux, J., et al. 1993, A\&A, 276, 25

Israel, F. P., Johansson, L. E. B., Rubio, M., et al. 2003, A\&A, 406, 817

Israel, F. P., Maloney, P. R., Geis, N., et al. 1996, ApJ, 465, 738

Keller, S. C., \& Wood, P. R. 2006, ApJ, 642, 834

Kennicutt, R. C., Jr. 1989, ApJ, 344, 685

Kennicutt, R. C., Jr. 1998, ApJ, 498, 541

Kennicutt, R. C., Jr., Bresolin, F., Bomans, D. J., Bothun, G. D., \& Thompson, I. B. 1995, AJ, 109, 594

Krumholz, M. R., Ellison, S. L., Prochaska, J. X., \& Tumlinson, J. 2009a, ApJ, 701, L12

Krumholz, M. R., Leroy, A. K., \& McKee, C. F. 2011, ApJ, 731, 25

Krumholz, M. R., \& McKee, C. F. 2005, ApJ, 630, 250

Krumholz, M. R., McKee, C. F., \& Tumlinson, J. 2009b, ApJ, 693, 216

Krumholz, M. R., McKee, C. F., \& Tumlinson, J. 2009c, ApJ, 699, 850

Kurt, C. M., Dufour, R. J., Garnett, D. R., et al. 1999, ApJ, 518, 246

Lada, C. J., Lombardi, M., \& Alves, J. F. 2009, ApJ, 703, 52

Leitherer, C., \& Heckman, T. M. 1995, ApJS, 96, 9

Lequeux, J., Le Bourlot, J., Pineau des Forets, G., et al. 1994, A\&A, 292, 371
Leroy, A., Bolatto, A., Stanimirović, S., et al. 2007a, ApJ, 658, 1027

Leroy, A., Cannon, J., Walter, F., Bolatto, A., \& Weiss, A. 2007b, ApJ, 663, 990

Leroy, A. K., Bolatto, A., Bot, C., et al. 2009, ApJ, 702, 352

Leroy, A. K., Bolatto, A. D., Gordon, K., et al. 2011, ApJ, 737, 12

Leroy, A. K., Walter, F., Brinks, E., et al. 2008, AJ, 136, 2782

Li, A., \& Draine, B. T. 2002, ApJ, 576, 762

Madden, S. C., Galliano, F., Jones, A. P., \& Sauvage, M. 2006, A\&A, 446, 877

Madden, S. C., Poglitsch, A., Geis, N., Stacey, G. J., \& Townes, C. H. 1997, ApJ, 483, 200

Maloney, P., \& Black, J. H. 1988, ApJ, 325, 389

Martin, C. L., \& Kennicutt, R. C., Jr. 2001, ApJ, 555, 301

Mathis, J. S., Mezger, P. G., \& Panagia, N. 1983, A\&A, 128, 212

McGee, R. X., \& Newton, L. M. 1981, Proc. Astron. Soc. Aust., 4, 189

McKee, C. F. 1989, ApJ, 345, 782

McKee, C. F., \& Ostriker, E. C. 2007, ARA\&A, 45, 565

Mizuno, N., Rubio, M., Mizuno, A., et al. 2001, PASJ, 53, L45

Müller, E., Ott, J., Hughes, A., et al. 2010, ApJ, 712, 1248

Nieten, C., Neininger, N., Guélin, M., et al. 2006, A\&A, 453, 459

Oliveira, J. M., van Loon, J. T., Sloan, G. C., et al. 2011, MNRAS, 411, L36

Ostriker, E. C., McKee, C. F., \& Leroy, A. K. 2010, ApJ, 721, 975

Pagel, B. E. J. 2003, in ASP Conf. Ser. 304, CNO in the Universe, ed. C. Charbonnel, D. Schaerer, \& G. Meynet (San Francisco, CA: ASP), 187

Planck Collaboration, Abergel, A., Ade, P., et al. 2011a, A\&A, submitted (arXiv:1101.2036)

Planck Collaboration, Ade, P. A. R., Aghanim, N., et al. 2011b, A\&A, submitted (arXiv:1101.2046)

Rafelski, M., Wolfe, A. M., \& Chen, H.-W. 2011, ApJ, 736, 48

Rahman, N., Bolatto, A. D., Wong, T., et al. 2011, ApJ, 730, 72

Relaño, M., Monreal-Ibero, A., Vílchez, J. M., \& Kennicutt, R. C. 2010, MNRAS, 402, 1635

Robertson, B. E., \& Kravtsov, A. V. 2008, ApJ, 680, 1083

Roychowdhury, S., Chengalur, J. N., Begum, A., \& Karachentsev, I. D. 2009, MNRAS, 397, 1435

Roychowdhury, S., Chengalur, J. N., Kaisin, S. S., Begum, A., \& Karachentsev, I. D. 2011, MNRAS, 414, L55

Rubin, D., Hony, S., Madden, S. C., et al. 2009, A\&A, 494, 647

Rubio, M., Lequeux, J., \& Boulanger, F. 1993, A\&A, 271, 9

Sandstrom, K. M., Bolatto, A. D., Draine, B. T., Bot, C., \& Stanimirović, S. 2010, ApJ, 715, 701

Sanduleak, N. 1969, AJ, 74, 47

Schaye, J. 2004, ApJ, 609, 667

Schaye, J., \& Dalla Vecchia, C. 2008, MNRAS, 383, 1210

Schmidt, M. 1959, ApJ, 129, 243

Schnee, S., Li, J., Goodman, A. A., \& Sargent, A. I. 2008, ApJ, 684, 1228

Schruba, A., Leroy, A. K., Walter, F., et al. 2011, AJ, 142, 37

Schruba, A., Leroy, A. K., Walter, F., Sandstrom, K., \& Rosolowsky, E. 2010, ApJ, 722, 1699

Smith, R. C. \& The MCELS Team. 1999, in IAU Symp. 190, New Views of the Magellanic Clouds, ed. Y.-H. Chu, N. Suntzeff, J. Hesser, \& D. Bohlender (Cambridge: Cambridge Univ. Press), 28

Stacey, G. J., Geis, N., Genzel, R., et al. 1991, ApJ, 373, 423

Stanimirović, S., Staveley-Smith, L., Dickey, J. M., Sault, R. J., \& Snowden, S. L. 1999, MNRAS, 302, 417

Stanimirović, S., Staveley-Smith, L., \& Jones, P. A. 2004, ApJ, 604, 176

Stanimirović, S., Staveley-Smith, L., van der Hulst, J. M., et al. 2000, MNRAS, 315,791

Szewczyk, O., Pietrzyński, G., Gieren, W., et al. 2009, AJ, 138, 1661

Tan, J. C. 2000, ApJ, 536, 173

Tumlinson, J., Shull, J. M., Rachford, B. L., et al. 2002, ApJ, 566, 857

van Loon, J. T., Oliveira, J. M., Gordon, K. D., Sloan, G. C., \& Engelbracht, C. W. 2010, AJ, 139, 1553

Walter, F., Cannon, J. M., Roussel, H., et al. 2007, ApJ, 661, 102

Wilke, K., Klaas, U., Lemke, D., et al. 2004, A\&A, 414, 69

Wolfe, A. M., \& Chen, H. 2006, ApJ, 652, 981

Wolfire, M. G., Hollenbach, D., \& McKee, C. F. 2010, ApJ, 716, 1191

Wolfire, M. G., Hollenbach, D., McKee, C. F., Tielens, A. G. G. M., \& Bakes, E. L. O. 1995, ApJ, 443, 152

Wolfire, M. G., McKee, C. F., Hollenbach, D., \& Tielens, A. G. G. M. 2003, ApJ, 587,278

Wong, T., \& Blitz, L. 2002, ApJ, 569, 157

Yoshizawa, A. M., \& Noguchi, M. 2003, MNRAS, 339, 1135

Young, J. S., Allen, L., Kenney, J. D. P., Lesser, A., \& Rownd, B. 1996, AJ, 112, 1903

Young, J. S., Schloerb, F. P., Kenney, J. D., \& Lord, S. D. 1986, ApJ, 304, 443

Young, J. S., \& Scoville, N. Z. 1991, ARA\&A, 29, 581 